\title{
A FLUID-CELL INTERACTION AND ADHESION ALGORITHM FOR TISSUE-COATING OF CARDIOVASCULAR IMPLANTS
}

\author{
JIAN HAO *, TSORNG-WHAY PAN ${ }^{\dagger}$, SUNČICA ČANIĆ $\ddagger$, ROLAND GLOWINSKI §, AND \\ DOREEN ROSENSTRAUCH, M.D., ๆ
}

\begin{abstract}
In this manuscript we develop a fluid-cell interaction and adhesion algorithm applied to modeling the cell coating of artificial surfaces of cardiovascular implants for improved biocompatibility. The fluid-cell interaction and adhesion algorithm proposed in this manuscript is a multi-scale model that captures the process of cell adhesion which occurs at a scale of a few nano meters, and couples it with fluid-cell interaction which occurs at a scale of a few hundred microns. Cells are assumed rigid and neutrally buoyant. Cell adhesion is modeled using a a kinetics-based dynamic adhesion algorithm. Fluid-cell interaction is modeled using the fictitious domain method with distributed Lagrange multipliers. The coupling between the two is achieved through a time-splitting scheme. Novel results regarding the influence of certain fluid dynamics parameters and adhesion parameters on the generation of a stable and strong tissue coating of artificial surfaces are presented. The modular nature of this algorithm makes it easily applicable to a large class of problems involving the processes of cell adhesion and fluid-cell interaction.
\end{abstract}

* Department of Mathematics, University of Houston, 4800 Calhoun Rd., Houston TX 77204-3008, USA jianh@math.uh.edu. Research supported in part by the NSF and NIH(NIGMS) under grant DMS-0443826, and by the Texas Higher Education Board under grant ARP-003652-0051-2006

†Department of Mathematics, University of Houston, 4800 Calhoun Rd., Houston TX 77204-3008, USA. Research supported in part by the NSF and NIH(NIGMS) under grant DMS-0443826, by the NSF under grant DMS 0209066 and by the University of Houston GEAR grant 2007-2008.

‡Department of Mathematics, University of Houston, 4800 Calhoun Rd., Houston TX 77204-3008, USA (canic@math.uh.edu). Research supported in part by the NSF under grants DMS-0337355 and DMS-0806941, by the NSF and NIH(NIGMS) under grant DMS-0443826, by the Texas Higher Education Board under grant ARP-003652-0051-2006 and by the University of Houston GEAR grant 2007-2008.

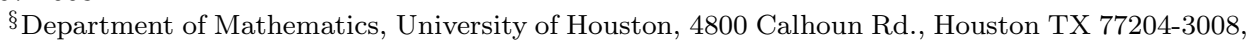
USA. Research supported in part by the NSF and NIH(NIGMS) grant DMS-0443826 and by the NSF under grant DMS-0412267.

IUniversity of Texas Health Science Center in Houston, and Texas Heart Institute at St. Luke's Episcopal Hospital, TX 77030, USA. Research supported in part by the NSF and NIH(NIGMS) under grant DMS-0443826 and by the Roderick Duncan MacDonald Foundation at St. Luke's Episcopal Hospital. 
1. A Brief Overview. In this manuscript we present a fluid-cell interaction and adhesion algorithm applied to modeling the cell coating of artificial surfaces of cardiovascular implants for improved biocompatibility.

The process of cell (tissue) coating involves seeding the cells on an artificial surface under conditions that promote cell adhesion. Cells are then exposed to fluid flowinduced shear stress. Fluid flow interacts with cells: those cells that are not firmly attached to the surface get detached due to the action of the hydrodynamic force, while cells that remain attached develop stable and stronger adhesion properties. The main goal of this manuscript is to develop the mathematical and computational tools to study the influence of certain parameters (e.g., the magnitude of shear stress, the cyclic loading due to oscillatory fluid flow, etc.) on the formation of a stable tissue coat. Details describing cell coating of cardiovascular implants are presented in Section 2 .

To study this problem we developed a fluid-cell interaction and adhesion algorithm. This is a multi-scale algorithm that captures the process of cell adhesion which occurs at a scale of a few nano meters, and couples it with fluid-cell interaction which occurs at a scale of a few hundred microns. Cells are assumed rigid and neutrally buoyant. Cell adhesion is modeled using a modification of a kinetics-based probabilistic algorithm developed by Dembo, Hammer et al. in [12, 20, 23]. Our adhesion algorithm is presented in Section 3.

The fluid flow is modeled by the Navier-Stokes equations for an incompressible, viscous fluid. The interaction between the fluid and cells is modeled using the fictitious domain/distributed Lagrange multipliers method, developed by Glowinski and Pan in $[17,29]$. The coupling between the adhesion algorithm and the fluid-particle interaction algorithm is achieved through a time-splitting scheme. The resulting method is modular in nature, making this algorithm applicable to a wide range of problems involving cell adhesion and fluid-particle interaction. Modeling additional features of the underlying problem, such as, for example, deformability of cells, can be easily incorporated in the proposed algorithm as a new module. A mathematical formulation of the fluid-particle interaction and adhesion algorithm together with the details of the numerical scheme, are presented in Section 4.

An investigation of the influence of certain hydrodynamics parameters and adhesion parameters on the formation of a stable tissue coating is presented in Section 5. In addition to the experimentally observed cell adhesion properties, our numerical investigation produced new information that is beyond the reach of experimental observations.

2. The Medical Application. Fluid-cell interaction and cell adhesion under controlled flow conditions play an important role in many physiological and bioengineering processes. They include adhesion of white blood cells to the arterial walls (endothelium) [7], tumor cell metastasis [35], binding of bacteria to the intestinal wall [37], adhesion of transplanted cartilage that resides in joints (articular cartilage) for injury repair [26, 33], and adhesion-based cell sorting used in biotechnological applications [15]. In this work we focus on a novel application: coating of artificial surfaces of endovascular devices such as stents, or left ventricular assist devices, with ear cartilage cells (auricular chondrocytes) for improved biocompatibility.

Stents are wire mesh tubes that serve as a scaffold to prop open the inside of a diseased artery, see Figure 2.1. One complication following the implantation of a stent in a coronary artery is restenosis (re-closure of an artery). In a sense, restenosis is scar tissue that forms in response to a mechanical intervention within a vascular 
structure with a foreign material of poor biocompatibility. To improve the biocom-
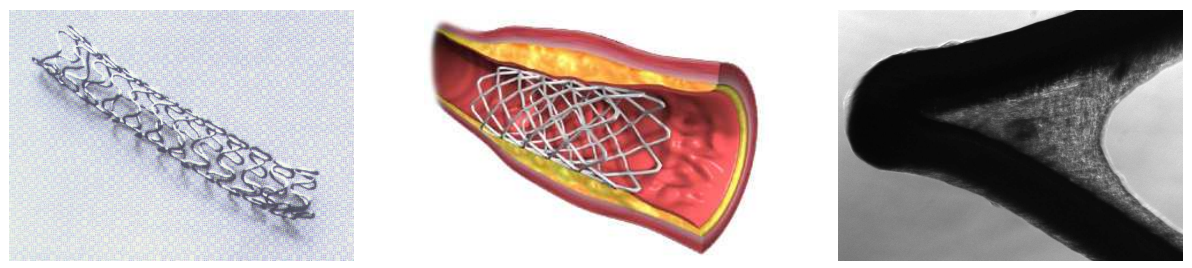

FIG. 2.1. Bare stent (left), stent inserted in a diseases artery (middle) and cartilage cell coated stent struts (magnification 1000X; day 8; results by D. Rosenstrauch MD, B. Magesa, M. Ng, D. Paniagua $M D$, D. Fish $M D$, OH Frazier $M D$ )

patibility of stents whose artificial surfaces will be contacting the blood stream, our group has begun investigating the ability of autologous auricular chondrocytes, i.e., ear cartilage cells, to form a strong adherent lining on artificial surfaces of stents [5]. Auricular cartilage harvested from the ear is abundantly available and easily accessible. Auricular chondrocytes have been shown to provide a strong adherent cell lining for left ventricular assist devices because of their ability to synthesize strong adherent extracellular matrix proteins [34]. Moreover, Rosenstrauch et al. have shown that it is possible to genetically engineer auricular chondrocytes to produce antithrombogenic factors (e.g., nitric oxide, prostacycline) [32]. This is a break-through discovery because seeding genetically engineered auricular chondrocytes onto artificial surfaces would result in the desired combination of tissue availability, strong surface adhesion, effective production of antithrombogenic factors and increased possibility of healing. Stents covered with genetically engineered ear cartilage cells might lower the restenosis rates and provide a long-lasting biocompatible prosthesis.

We have recently begun producing a coronary stent lined with chondrocytes. Ear cartilage cells were seeded onto a stent placed in a cell culture medium supplemented with vitamin $\mathrm{C}$ for improved collagen synthesis. Figure 2.1 (right) shows stent struts after three days of being immersed in the cell culture medium, covered with substantial amounts of extracellular matrix components which cover the small pores in the stent and extend between the struts showing inclination for complete prosthesis coverage. A mathematical and experimental model of growth of ear cartilage on artificial surfaces, developed by Čanić and Rosenstrauch in [4], provides information about optimal seeding strategy for fastest surface coverage.

The second phase in the production of a tissue-coated stent involves pre-conditioning of the tissue coat by exposing the tissue to mechanical loading induced by fluid shear stress. It has been experimentally observed in [34] that at the beginning stages of chondrocyte exposure to fluid shear stress, a certain percentage of cells detach. At the same time the strength and type of adhesion changes [33, 39]. The adhesion becomes stronger and the percentage of cells lost diminishes, leading to a stable tissue configuration.

In this manuscript we investigate how certain hydrodynamic parameters and adhesion parameters influence the formation of a stable and strong tissue coating. The parameters we consider are the flow shear rate, the type of shear flow (linear vs. oscillatory), the length of exposure to the fluid flow-induced shear stress, the number of adhesion molecules covering the cells, the number of seeded cells and the initial distribution of seeded cells. We present a novel computational scheme for a multi-scale model approximating auricular chondrocyte adhesion and detachment under shear 
flow conditions with the purpose of modeling stable tissue formation for coating of artificial surfaces of endovascular devices.

3. Cell Adhesion. Cellular adhesion is the binding of a cell to another cell or to a non-cellular component such as a surface or an extracellular matrix. Cell adhesion is regulated by specific cell adhesion molecules called receptors that react with molecules on the opposing cell or surface, called ligands.

Cell adhesion molecules (CAMs) are proteins located on the surface of a cell. Most of the CAMs belong to the following four protein families: the integrins, the selectins, the cadherins and the immunoglobulin superfamily.

Cell adhesion is a highly complex, dynamic, multi-stage process. The initial stage of cell adhesion to external surfaces is mediated, for certain cells including chondrocytes, by cell-surface hyaluronan (a glycosaminoglycan which is present as a coat around each chondrocyte) $[9,39,40]$. This stage is quickly followed by a stronger, integrin-mediated adhesion which begins with the dot-shaped focal complexes and then changes to focal adhesion [39]. It was reported in [39] that the hyaluronanmediated adhesion is replaced by the integrin-mediated adhesion within a few tens of seconds to minutes. Continuous application of force, such as the fluid flow-induced shear stress, results in the formation of fibrillar adhesion and reorganization of the extracellular matrix [39]. Fibrillar adhesion binds a certain cell integrin with the extracellular matrix fibronectin fibrils giving rise to the strongest type of adhesion in this multi-stage adhesion process.

Adhesion of auricular chondrocytes and their behavior under shear flow has not been studied extensively in literature. Since auricular chondrocytes naturally reside in the ear, the number of studies relating adhesion of auricular chondrocytes to artificial surfaces and fluid flow-induced shear stress is rather small. The most useful to our research have been the works by Scott-Burden et al. in [34] and by Chung et al. in [10]. In the work reported in [34] cells were seeded on the endoluminal surface of a Left Ventricular Assist Device (LVAD) for 24 hours, and then left 4 days in an incubator to promote the extracellular matrix synthesis. The tissue was then exposed to fluid flow-induced shear stress by including the LVAD in a flow loop. At the initial stages of tissue exposure to shear stress a number of cells detached. The percentage of detached cells versus time formed a linear function. As the time progressed, the percentage of detached cells tapered off, until it reached a steady state of $11 \%$ detachment. After the implantation of the LVAD into a calf for 7 days, none of the organs showed any evidence of emboli, ischemia or infarction, indicating the formation of a stable and well-adhered tissue coating. We will be using these experimental observations reported in [34] as a guide for the design of our mathematical model to capture the cell loss and formation of a stable tissue coating for cardiovascular implants.

3.1. A Kinetics Model of Cell Adhesion. There has been extensive work in the literature on modeling and simulation of cell adhesion to a substrate mediated by receptor-ligand interactions. Mathematical models proposed so far to describe different events in cell adhesion are based on either the equilibrium concept [2] or the kinetics concept $[6,12,23,25]$. See [28] for a nice overview. In our work we follow the kinetics-based probabilistic Dynamic Adhesion Algorithm by Hammer et al. [6, 23, 25] used to study leukocyte rolling and adhesion to the endothelium in Stokes flow. In their algorithm, a cell is treated as a rigid ball and the kinetics of bond association and dissociation is modeled in a probabilistic fashion. There are computational models that include deformability of cells by using either the elastic ring model [13, 14] or a compound drop model [28]. These are two-dimensional models for deformable 
cells with the kinetics of receptor-ligand bonds represented by deterministic relations. Jadhav et al. developed a three-dimensional computational model for deformable cells based on the immersed boundary method [21]. Reboux et al. introduced bond tilting in their 2D model in which a cell is considered as a rigid cylinder with the bonds that can stretch and tilt [31].

We have decided to adopt the approach of Hammer et al. [6, 23, 25] in our application to study cell pre-conditioning for auricular chondrocytes, because this algorithm can handle the different stages of dynamic cell adhesion, and our modifications based on a dynamic conversion of the probabilistic algorithm to a deterministic one, appear to be able to capture the formation of a stable auricular cartilage coating of artificial surfaces observed in experiments performed at the Texas Heart Institute [34].

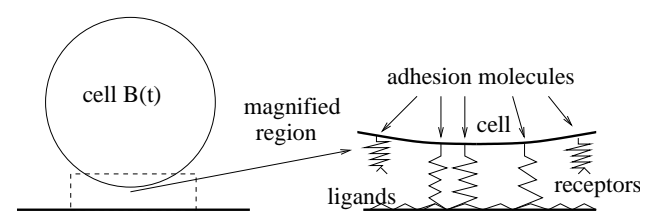

FIG. 3.1. Model of receptor-ligand mediated cell adhesion to a substrate. The bottom surface is covered by ligands and the cell surface is covered by randomly distributed receptors.

In the probabilistic Dynamic Adhesion Algorithm [6, 23, 25] the adhesion molecules are modeled as linear, Hookean springs, distributed randomly over the cell surface as shown in Figure 3.1. Bond association or dissociation occur according to the forward reaction rate, $k_{f}$, and the reverse reaction rate, $k_{r}$, respectively. The forward and reverse reaction rates, derived in [12], are functions of the separation distance between the cell and the surface. If we characterize each bond by a time-dependent vector $\mathbf{x}_{b}$, then the separation distance is the length of $\mathbf{x}_{b}$, denoted by $\left|\mathbf{x}_{b}\right|$. The proposed reaction rates in [12] are given by:

$$
\begin{gathered}
k_{f}\left(\left|\mathbf{x}_{b}\right|\right)=k_{f}^{0} \exp \left(-\frac{\sigma_{t s}\left(\left|\mathbf{x}_{b}\right|-\lambda\right)^{2}}{2 k_{b} T}\right), \\
k_{r}\left(\left|\mathbf{x}_{b}\right|\right)=k_{r}^{0} \exp \left(\frac{\left(\sigma-\sigma_{t s}\right)\left(\left|\mathbf{x}_{b}\right|-\lambda\right)^{2}}{2 k_{b} T}\right),
\end{gathered}
$$

where the constants appearing in these expressions are the following: $\lambda$ is the equilibrium spring length, $\sigma$ is the spring constant (of an established bond), and $\sigma_{t s}$ is the spring constant of the transition state. Here it was assumed that the difference between the transition state and the bonded state can be described by a change in the spring constant only. Furthermore, $k_{b}$ is the Boltzmann constant, and $T$ is the absolute temperature, and $k_{f}^{0}$ and $k_{f}^{0}$ are the forward and the reverse reaction rates, respectively, when the spring length $\left|\mathbf{x}_{b}\right|$ is at its equilibrium length $\lambda$. The ratio of the forward reaction rate and the reverse reaction rate at any separation distance satisfies:

$$
\frac{k_{f}}{k_{r}}=\frac{k_{f}^{0}}{k_{r}^{0}} \exp \left(-\frac{\sigma\left(\left|\mathbf{x}_{b}\right|-\lambda\right)^{2}}{2 k_{b} T}\right) .
$$

This equation states that the likelihood of finding an established bond decreases as the separation distance deviates from equilibrium. 
The probabilistic Dynamic Adhesion Algorithm models bond formation and breakage as a function of time. The time interval is divided into subintervals of length $\Delta t$. Given a time interval $\Delta t$, the probability of the formation of a new bond is given by the following probability of binding at the end of the time interval $\Delta t$

$$
P_{f}=1-\exp \left(-k_{f} \Delta t\right),
$$

where $k_{f}$ is the forward reaction rate. Our algorithm, based on a random number generator, associates a receptor-ligand bond if the generated random number is less than $P_{f}$.

Similarly, the probability that a bond which already existed prior to the current time interval will break during the time interval $\Delta t$ is given by

$$
P_{r}=1-\exp \left(-k_{r} \Delta t\right),
$$

where $k_{r}$ is the reverse reaction rate. If the generated random number is less than $P_{r}$ the bond breaks at this time step.

Once a bond is established, the force imparted by the spring representing the bond is given by

$$
\mathbf{F}_{b}=\sigma\left(\left|\mathbf{x}_{b}\right|-\lambda\right) \mathbf{u}_{b}
$$

where $\mathbf{u}_{b}=\mathbf{x}_{b} /\left|\mathbf{x}_{b}\right|$ is the unit vector associated with $\mathbf{x}_{b}$.

The sum over all established bonds gives the total adhesion force $\mathbf{F}^{a}$ imparted on a cell. If $E(t)$ denotes the number of established bonds at time $t$, then the total adhesion force $\mathbf{F}^{a}(t)$ equals

$$
\mathbf{F}^{a}(t)=\sum_{b=1}^{E(t)} \mathbf{F}_{b}=\sum_{b=1}^{E(t)} \sigma\left(\left|\mathbf{x}_{b}\right|-\lambda\right) \mathbf{u}_{b}
$$

Force $\mathbf{F}^{a}$ will be used in the next section to calculate the fluid-cell interactions for adhered cells. Cells exposed to fluid shear stress will move depending on the total force exerted on each cell, which is a resultant of the hydrodynamic forces, the total adhesion force acting on each cell, and the short-range repulsion force used to model collisions and prevent overlap of rigid bodies.

By combining this Dynamic Adhesion Algorithm with the fluid-particle interaction algorithm which will be described in Section 4, we performed several computational investigations to simulate the formation of a strong and stable adhesion of auricular chondrocytes, reported in [34]. To capture the transition to strong (fibrillar) adhesion we modified the spring constant so that it increased as a function of time. This is in addition to taking $\sigma_{t s}>\sigma$ so that $k_{r}\left(\left|x_{b}\right|\right) \rightarrow 0$ as $\left|\mathbf{x}_{b}\right| \rightarrow 0$, describing the "catch bonds" as opposed to the "slip bonds", see [12]. Regardless of how large the spring constant was, the probabilistic adhesion algorithm led to cell rolling and cell loss that continued indefinitely. This is why we decided to test a modification of the probabilistic Dynamic Adhesion Algorithm that would include a continuous, time-dependent conversion of the probabilistic adhesion into a deterministic one. This produced results that we were looking for: no cell rolling and steady cell-detachment rate after a certain time, as observed in experiments. This algorithm modification is presented next. 
3.2. A Modified Dynamic Adhesion Algorithm. To capture the observed adhesive properties of auricular chondrocytes exposed to fluid flow-induced shear stress [34], we introduce two modifications of the Dynamic Adhesion Algorithm presented in $[6,23,25]$. Both modifications are related to the changes over time in the adhesion properties of auricular chondrocytes. These changes have been implemented by modifying certain model constants to become functions of time in a way which does not change the ratio between the forward and reverse reaction rates:

1. The spring constants $\sigma$ and $\sigma_{t s}$ become increasing functions of time;

2. The probabilistic kinetics of bond association and dissociation switches to a deterministic one, continuously over time, with the equilibrium reaction rates decreasing over time. In the deterministic algorithm an already established bond breaks if the spring length $\left|\mathbf{x}_{b}\right|$ becomes longer than a given value.

These modifications were implemented in the following way:

1. The spring constants $\sigma\left(\sigma_{t s}\right)$ are replaced by the product of $\sigma\left(\sigma_{t s}\right)$ and a function $q_{1}(t)$, where $q_{1}(t)$ is a monotonically increasing function, bounded as $t \rightarrow \infty$. In our numerical implementation we used

$$
q_{1}(t)=\frac{10}{1+(10-1) e^{-\omega_{0} t}} \text { with } \omega_{0}=20 s^{-1} .
$$

2. The reaction rates $k_{f}^{0}\left(k_{r}^{0}\right)$ are replaced by the product of $k_{f}^{0}\left(k_{r}^{0}\right)$ and a function $q_{2}(t)$, where $q_{2}(t)$ is given by a monotonically decreasing function approaching 0 , as $t \rightarrow \infty$. In our numerical implementation we used

$$
q_{2}(t)=2-\frac{2}{1+(2-1) e^{-\omega_{0} t}} \text { with } \omega_{0}=20 s^{-1} .
$$

Clearly, the ratio of the reaction rates remains the same.

4. Fluid-Particle Interaction and Adhesion Algorithm. In this section we discuss a distributed Lagrange multiplier-based fictitious domain method for the simulation of fluid-particle interaction between neutrally buoyant cells adhering to an artificial surface, exposed to the Newtonian fluid flow modeled by the Navier-Stokes equations. The fluid-particle interaction algorithm adopted in this work is based on the works by Glowinski, Pan et al, presented in [16, 17, 18, 29]. The implementation of the method in this manuscript utilizes a time-splitting scheme. The time splitting scheme for the Lagrange multiplier-based fictitious domain method is particularly suitable for the use in our application for the following three reasons:

1. The algorithm uses a simple structured mesh instead of a boundary fitted mesh following the position of each particle. Particle motion is tracked by employing geometrical relations between a particle and the mesh. This substantially reduces computational complexity of the algorithm and is the main advantage of the fictitious domain method.

2. The hydrodynamic forces and torque imposed onto the rigid particles by the fluid do not need to be calculated explicitly since they are built in the global variational formulation implicitly. The rigid body motion of each particle is enforced via a Lagrange multiplier approach.

3. The dynamic adhesion algorithm can easily be added as a "nano-scale module". The coupling between the nano-scale adhesion dynamics module and the micro-scale fluid-particle interaction solver is done via passing the information about particle location from the micro- to the nano-scale solver and 
then passing the information about the total particle adhesion force from the nano- to the micro-scale. This is performed through a particular time-splitting method.

4.1. The Mathematical Model. Let $\Omega \subset \mathbb{R}^{n}$ where $n=2$, be a rectangular domain with boundary $\Gamma$. Points of $\Omega$ will be denoted by $\mathbf{x}=\left(x_{1}, x_{2}\right)$. The domain $\Omega$ is filled with fluid and particles. See Figure 4.1. The size of $\Omega$ is at the order of a few hundred microns, while the cell size is a few microns. The adhesion process takes place at the scale of a few nano-meters. We assume that the fluid is a Newtonian

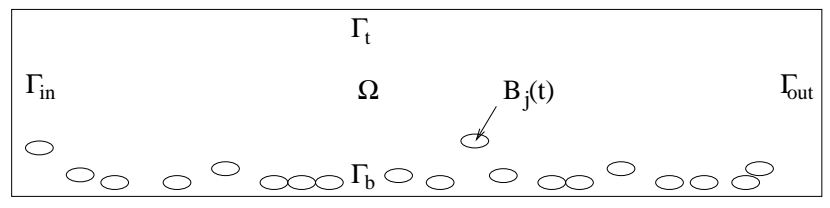

FIG. 4.1. A sketch of the domain $\Omega$ containing an incompressible viscous fluid with immersed cells $B_{j}(t), j=1, \ldots, J$.

viscous incompressible fluid of density $\rho_{f}$ and viscosity $\mu_{f}$. The particles are assumed to be neutrally buoyant, i.e., of density $\rho_{f}$, and rigid. The region in $\mathbb{R}^{2}$ occupied by each particle is denoted by $B_{j}(t), j=1, \ldots, J$ and the center of mass of each particle is denoted by $\mathbf{G}_{j}(t)=\left(\left(G_{j}\right)_{1}(t),\left(G_{j}\right)_{2}(t)\right)^{t}$ at time $t$. The fluid flow is modeled by the Navier-Stokes equations. If we denote by $B(t)$ the union of all the particles, $B(t)=\cup_{j=1}^{J} B_{j}(t)$, then the fluid flow equations can be written as:

$$
\begin{array}{r}
\rho_{f}\left[\frac{\partial \mathbf{u}}{\partial t}+(\mathbf{u} \cdot \nabla) \mathbf{u}\right]=\mathbf{F}+\nabla \cdot \boldsymbol{\sigma}, \quad \text { in } \Omega \backslash \overline{B(t)}, \\
\nabla \cdot \mathbf{u}=0, \quad \text { in } \Omega \backslash \overline{B(t),}
\end{array}
$$

where $\mathbf{u}$ and $p$ denote the fluid velocity and pressure, respectively, $\mathbf{F}$ is the body force, and the fluid stress tensor $\boldsymbol{\sigma}$ is given by

$$
\boldsymbol{\sigma}=2 \mu_{f} \mathbf{D}(\mathbf{u})-p \mathbf{I},
$$

with $\mathbf{D}(\mathbf{v})=\left[\boldsymbol{\nabla} \mathbf{v}+(\boldsymbol{\nabla} \mathbf{v})^{t}\right] / 2$ denoting the symmetrized gradient of velocity. The initial condition is given by

$$
\mathbf{u}(\mathbf{x}, 0)=\mathbf{u}_{0}(\mathbf{x}), \forall \mathbf{x} \in \Omega \backslash \overline{B(t)}, \text { with } \nabla \cdot \mathbf{u}_{0}=0 .
$$

The boundary $\Gamma$ consists of the top and bottom parts $\Gamma_{t}$ and $\Gamma_{b}$ respectively, and the "inlet" and "outlet" part $\Gamma_{\text {in }}$ and $\Gamma_{\text {out }}$. We prescribe periodic boundary conditions at the inlet and outlet boundary, zero velocity at the bottom boundary, and the following boundary condition at the top boundary:

$$
\mathbf{u}=\mathbf{g}_{0}(t)=\left(g_{0}(t), 0\right)^{t}, \quad \text { on } \Gamma_{t} .
$$

This set of boundary conditions on $\Gamma$ satisfies the requirement that the total integral over $\Gamma$ of the boundary velocity projected onto the outer normal to the boundary, be equal to zero. This is consistent with the incompressibility condition and the assumption that the particles are rigid, namely that $\int_{\partial B_{j}} \mathbf{u} \cdot \mathbf{n} d\left(\partial B_{j}\right)=0$.

At the boundary of each ball $B_{j}(t)$ we assume the no-slip boundary condition:

$$
\mathbf{u}(\mathbf{x}, t)=\mathbf{V}_{j}(t)+\boldsymbol{\omega}_{j}(t) \times\left(\mathbf{x}-\mathbf{G}_{j}(t)\right), \quad \forall \mathbf{x} \in \partial B_{j}(t),
$$


where $\mathbf{V}_{j}$ denotes the velocity of the center of mass $\mathbf{G}_{j}$ (translation velocity), and $\boldsymbol{\omega}_{j}$ is the angular velocity of the $j$-th body. For our two-dimensional problem, $\boldsymbol{\omega}_{j}=$ $\left(0,0, \omega_{j}\right)$, and so we can write $\boldsymbol{\omega}_{j}(t) \times\left(\mathbf{x}-\mathbf{G}_{j}(t)\right)=\omega_{j}\left(\mathbf{x}-\mathbf{G}_{j}\right)^{\perp}$ where $\left(\mathbf{x}-\mathbf{G}_{j}\right)^{\perp}=$ $\left(-\left(x_{2}-\left(G_{j}\right)_{2}\right), x_{1}-\left(G_{j}\right)_{1}\right)^{t}$. Thus, the no-slip condition in $n=2$ reads

$$
\mathbf{u}(\mathbf{x}, t)=\mathbf{V}_{j}(t)+\omega_{j}\left(\mathbf{x}-\mathbf{G}_{j}\right)^{\perp}, \quad \forall \mathbf{x} \in \partial B_{j}(t) \text { and } n=2 .
$$

The translation velocity satisfies

$$
\frac{d \mathbf{G}_{j}}{d t}=\mathbf{V}_{j}
$$

The motion of each particle is modeled by the Newton-Euler equations describing the balance of linear and angular momentum for each particle

$$
\begin{array}{r}
M_{j} \frac{d \mathbf{V}_{j}}{d t}=M_{j} \mathbf{g}+\mathbf{F}_{j}^{h}+\mathbf{F}_{j}^{a}+\mathbf{F}_{j}^{r}, \\
\mathbf{I}_{j} \frac{d \boldsymbol{\omega}_{j}}{d t}+\boldsymbol{\omega}_{j} \times \mathbf{I}_{j} \boldsymbol{\omega}_{j}=\mathbf{T}_{j},
\end{array}
$$

where $M_{j}$ is the mass of the $j$ th rigid body, $\mathbf{g}$ is the acceleration due to gravity, $\mathbf{I}_{j}$ is the inertia tensor of the $j$ th body, $\mathbf{F}_{j}^{h}$ is the resultant of the hydrodynamic forces acting on the $j$ th body, $\mathbf{F}_{j}^{a}$ is the resultant of the adhesion forces acting on the $j$ th body, $\mathbf{F}_{j}^{r}$ is the short-range repulsive force, and $\mathbf{T}_{j}$ is the torque at $\mathbf{G}_{j}$ of the hydrodynamic forces acting on the $j$ th body.

The short-range repulsive force is used to model collisions and prevent overlap by the regions occupied by the rigid bodies. As in [23], we will be assuming that a short-range repulsive force between two rigid bodies is equal to

$$
\mathbf{F}^{r}(d)=\mathbf{F}_{0} \frac{1}{d} \frac{e^{-\epsilon / d}}{1-e^{-\epsilon / d}},
$$

where $d$ is a length scale and $\epsilon$ is the separation. In the case of cell-boundary interactions $\mathbf{F}^{r}$ is directed normal to the plane. In the case of cell-cell interactions $\mathbf{F}^{r}$ is directed along the line connecting the centers of cells. In our case, the short-range repulsive force gets activated once the distance between two rigid bodies becomes smaller than the equilibrium spring length $\lambda$.

The following initial conditions supplement the initial-value problem for the NewtonEuler equations:

$$
B_{j}(0)=B_{0, j}, \mathbf{G}_{j}(0)=G_{0, j}, \mathbf{V}_{j}(0)=\mathbf{V}_{0, j}, \boldsymbol{\omega}_{j}(0)=\boldsymbol{\omega}_{0, j}, \forall j=1, \ldots, J .
$$

For our two-dimensional problem the nonlinear term $\boldsymbol{\omega}_{j} \times \mathbf{I}_{j} \boldsymbol{\omega}_{j}$ in the Newton-Euler equations (4.8) vanishes, and so the Newton-Euler equations in two dimensions read

$$
\left.\begin{array}{c}
M_{j} \frac{d \mathbf{V}_{j}}{d t}=M_{j} \mathbf{g}+\mathbf{F}_{j}^{h}+\mathbf{F}_{j}^{a}+\mathbf{F}_{j}^{r}, \\
\mathbf{I}_{j} \frac{d \boldsymbol{\omega}_{j}}{d t}=\mathbf{T}_{j},
\end{array}\right\} \text { for } n=2 .
$$

4.2. Time-discretization by operator splitting. We approximate problem (4.1)-(4.10) in time by using Lie's scheme [8]. Lie's scheme can be summarized as follows. Consider the following initial-value problem

$$
\begin{aligned}
\frac{\partial \phi}{\partial t}+A(\phi) & =0 \text { in }(0, T), \\
\phi(0) & =\phi_{0}, \\
& 9
\end{aligned}
$$


where $A$ is a (nonlinear) operator from a Hilbert space to itself. Suppose that operator $A$ has a non-trivial decomposition

$$
A=\sum_{i=1}^{I} A_{i}
$$

Then, the solution of (4.11) can be approximated in time by the solution of the following scheme. Let $\Delta t>0$ be a time-discretization step. Denote $t^{n}=n \Delta t$ and let $\phi^{n}$ be an approximation of $\phi\left(t^{n}\right)$. Set $\phi^{0}=\phi_{0}$. Then, for $n \geq 0$ compute $\phi^{n+1}$ by solving

$$
\begin{aligned}
\frac{\partial \phi_{i}}{\partial t}+A_{i}\left(\phi_{i}\right) & =0 \text { in }\left(t_{n}, t_{n+1}\right), \\
\phi_{i}\left(t^{n}\right) & =\phi^{n+(i-1) / I} ; \phi^{n+i / I}=\phi_{i}\left(t^{n+1}\right),
\end{aligned}
$$

for $i=1, \ldots, I$. This method is first-order accurate. More precisely, if (4.11) is defined on a finite-dimensional space and if the operators $A_{i}$ are smooth enough, then $\left\|\phi\left(t^{n}\right)-\phi^{n}\right\|=O(\Delta t)$.

We split problem (4.1)-(4.10) by using Lie's scheme in order to deal with cell adhesion and collisions separately from fluid-particle interaction. This is done in a "predictor-corrector" fashion:

1. The Newton-Euler equations are solved for the velocity of each particle with only one half of the adhesion and repulsion force contributions $\left(M_{j} d \mathbf{V}_{j} / d t=\right.$ $\left.\left(\mathbf{F}_{j}^{a}+\mathbf{F}_{j}^{r}\right) / 2\right)$; equation (4.6) is solved for the position of each particle by taking into account the contribution of one half of the velocity $\left(d \mathbf{G}_{j} / d t=\mathbf{V}_{j} / 2\right)$ with appropriate initial conditions;

2. The fluid-particle interaction problem is solved by accounting for the contribution of the hydrodynamic force and gravity in the Newton-Euler equations $\left(M_{j} d \mathbf{V}_{j} / d t=\mathbf{F}_{j}^{h}+M_{j} \mathbf{g}\right)$ and the contribution due to torque $\left(\mathbf{I}_{j} d \boldsymbol{\omega}_{j} / d t=\mathbf{T}_{j}\right)$; the velocity of each particle is updated;

3. The final position of each particle and translation velocity are obtained by solving the Newton-Euler equations for the velocity of each particle with the remaining one half of the adhesion and repulsion force contributions $\left(M_{j} d \mathbf{V}_{j} / d t=\left(\mathbf{F}_{j}^{a}+\mathbf{F}_{j}^{r}\right) / 2\right)$ and by solving equation (4.6) with the contribution of the remaining one half of the translation velocity $\left(d \mathbf{G}_{j} / d t=\mathbf{V}_{j} / 2\right)$ and with appropriate initial conditions.

The fluid-particle interaction problem described in Step 2 is solved by using a fictitious domain method with distributed Lagrange multipliers.

4.3. A Distributed Lagrange Multiplier-Based Fictitious Domain Formulation for the Fluid-Particle Interaction Sub-Problem. A distributed Lagrange multiplier-based fictitious domain method for the simulation of fluid-particle interactions with rigid particles that are not necessarily neutrally buoyant, was first introduced in [17]. The neutrally buoyant case was considered in [30]. We summarize the main steps next.

The fictitious domain method is based on extending the problem defined on $\Omega \backslash \overline{B(t)}$ onto the entire domain $\Omega$ by assuming that each rigid particle is filled with the surrounding fluid and that it satisfies the rigid body motion constraints. This means, among other things, that the velocity field $\mathbf{u}$ satisfies $\nabla \cdot \mathbf{u}=0$, and $\mathbf{D}(\mathbf{u})=0$. To derive a weak formulation of the problem, we use a standard approach: we multiply the Navier-Stokes equations by a test function and integrate by parts. The fluid stress 
contributes to the motion of rigid particles through the boundary terms on $\partial B_{i}(t)$, $i=1, \ldots, J$. The Newton-Euler equations incorporating the hydrodynamic force and the torque, are used to couple the influence of hydrodynamic stress to the motion of rigid particles. The integrals are then extended appropriately to the entire domain by taking into account the fact that our particles are neutrally buoyant. For more details see $[17,29,30]$. The resulting global weak formulation is given by the following.

Define the following function spaces:

$$
\begin{aligned}
& W_{\mathbf{g}_{0}, p}=\left\{\mathbf{v} \mid \mathbf{v} \in\left(H^{1}(\Omega)\right)^{2}, \mathbf{v}=\mathbf{g}_{0}(t) \text { on } \Gamma_{t}, \mathbf{v}=\mathbf{0} \text { on } \Gamma_{b},\right. \text { and } \\
& \quad \begin{array}{r}
\left.\mathbf{v} \text { is periodic in the } x_{1} \text { direction }\right\} \\
W_{0, p}=\left\{\mathbf{v} \mid \mathbf{v} \in\left(H^{1}(\Omega)\right)^{2}, \mathbf{v}=\mathbf{0} \text { on } \Gamma_{t} \cup \Gamma_{b}\right. \text { and } \\
\left.\quad \mathbf{v} \text { is periodic in the } x_{1} \text { direction }\right\}
\end{array} \\
& L_{0}^{2}=\left\{q \mid q \in L^{2}(\Omega), \int_{\Omega} q d \mathbf{x}=0\right\}
\end{aligned}
$$

and the spaces of Lagrange multipliers, $\Lambda_{0}^{j}(t)$ for $j=1, \ldots, J$, which are going to be used to relax the rigid body motion constraint (4.5):

$$
\Lambda_{0}^{j}(t)=\left\{\boldsymbol{\mu}_{j} \mid \boldsymbol{\mu}_{j} \in\left(H^{1}\left(B_{j}(t)\right)\right)^{2},<\boldsymbol{\mu}_{j}, \mathbf{e}_{i}>_{j}=0, i=1,2,<\boldsymbol{\mu}_{j},\left(\mathbf{x}-\mathbf{G}_{j}\right)^{\perp}>_{j}=0\right\} .
$$

Here $\mathbf{e}_{1}=\{1,0\}^{t}, \mathbf{e}_{2}=\{0,1\}^{t},\left(\mathbf{x}-\mathbf{G}_{j}\right)^{\perp}=\left\{-\left(x_{2}-\left(G_{j}\right)_{2}\right), x_{1}-\left(G_{j}\right)_{1}\right\}^{t}$ and $<\cdot, \cdot>_{j}$ denotes an inner product on $H^{1}\left(B_{j}(t)\right)^{2}$ which can be defined as, for example,

$$
<\boldsymbol{\mu}, \mathbf{v}>_{j}=\int_{B_{j}(t)}\left(\boldsymbol{\mu} \cdot \mathbf{v}+\delta_{j}^{2} \mathbf{D}(\boldsymbol{\mu}): \mathbf{D}(\mathbf{v})\right) d \mathbf{x},
$$

where $\delta_{j}$ is the characteristic length (the diameter of $B_{j}$, for example) (see [17], Section 5 , for further information on the choices of $<\cdot, \cdot\rangle_{B_{j}}$ ).

The following is a fictitious domain formulation with Lagrange multipliers for the fluid-particle interaction problem:

For a.a. $t>0$, find $\mathbf{u}(t) \in W_{\mathbf{g}_{0}, p}, p(t) \in L_{0}^{2}, \mathbf{V}_{j}(t) \in \mathbb{R}^{2}, \mathbf{G}_{j}(t) \in \mathbb{R}^{2}$, $\omega_{j}(t) \in \mathbb{R}, \boldsymbol{\lambda}_{j}(t) \in \Lambda_{0}^{j}(t)$ such that

$$
\begin{aligned}
& \left\{\begin{array}{l}
\rho_{f} \int_{\Omega}\left[\frac{\partial \mathbf{u}}{\partial t}+(\mathbf{u} \cdot \boldsymbol{\nabla}) \mathbf{u}\right] \cdot \mathbf{v} d \mathbf{x}+2 \mu_{f} \int_{\Omega} \mathbf{D}(\mathbf{u}): \mathbf{D}(\mathbf{v}) d \mathbf{x}-\int_{\Omega} p \boldsymbol{\nabla} \cdot \mathbf{v} d \mathbf{x} \\
\quad-\sum_{j=1}^{J}<\boldsymbol{\lambda}_{j}, \mathbf{v}>_{j}=\int_{\Omega} \mathbf{F} \cdot \mathbf{v} d \mathbf{x}, \forall \mathbf{v} \in W_{0, p}
\end{array}\right. \\
& \int_{\Omega} q \boldsymbol{\nabla} \cdot \mathbf{u}(t) d \mathbf{x}=0, \forall q \in L^{2}(\Omega) \\
& <\boldsymbol{\mu}_{j}, \mathbf{u}(t)>_{j}=0, \forall \boldsymbol{\mu}_{j} \in \Lambda_{0}^{j}(t), \forall j=1, \ldots, J \\
& \frac{d \mathbf{G}_{j}}{d t}=\mathbf{V}_{j}, j=1, \ldots, J \\
& \mathbf{V}_{j}(0)=\mathbf{V}_{j}^{0}, \omega_{j}(0)=\omega_{j}^{0}, \mathbf{G}_{j}(0)=\mathbf{G}_{j}^{0}=\left\{\left(G_{j}^{0}\right)_{1},\left(G_{j}^{0}\right)_{2}\right\}^{t} \\
& \mathbf{u}(\mathbf{x}, 0)=\overline{\mathbf{u}}_{0}(\mathbf{x})=\left\{\begin{array}{l}
\mathbf{u}_{0}(\mathbf{x}), \forall \mathbf{x} \in \Omega \backslash \overline{B(0)} \\
\mathbf{V}_{j}^{0}+\omega_{j}^{0}\left\{-\left(x_{2}-\left(G_{j}^{0}\right)_{2}\right), x_{1}-\left(G_{j}^{0}\right)_{1}\right\}^{t}, \forall \mathbf{x} \in \overline{B_{j}(0), \forall j}
\end{array}\right.
\end{aligned}
$$

Here, $\mathbf{F}$ is the pressure gradient pointing in the $x_{1}$ direction. 


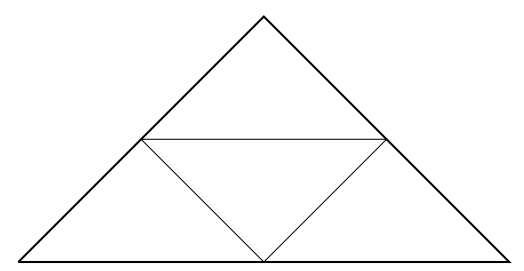

FIG. 4.2. Subdivision of a triangle of $\mathcal{T}_{2 h}$.

To recover the translation velocity $\mathbf{V}_{j}$ and angular speed $\omega_{j}(t)$ of each particle $B_{j}$, we solve the following equations for each $j=1, \ldots, J$ :

$$
\left\{\begin{array}{l}
<\mathbf{e}_{i}, \mathbf{u}(t)-\mathbf{V}_{j}(t)-\omega_{j}(t)\left(\mathbf{x}-\mathbf{G}_{j}\right)^{\perp}>_{j}=0, \text { for } i=1,2, \\
<\left(\mathbf{x}-\mathbf{G}_{j}\right)^{\perp}, \mathbf{u}(t)-\mathbf{V}_{j}(t)-\omega_{j}(t)\left(\mathbf{x}-\mathbf{G}_{j}\right)^{\perp}>_{j}=0 .
\end{array}\right.
$$

REMARK 4.1. The hydrodynamic forces and torque imposed on the rigid body by the fluid are built in the weak formulation (4.15)-(4.20) implicitly (see [16, 17] for details). Since in (4.15)-(4.20) the flow field is defined on the entire domain $\Omega$, it can be computed with a simple structured grid.

REMARK 4.2. In (4.15), $2 \int_{\Omega} \mathbf{D}(\mathbf{u}): \mathbf{D}(\mathbf{v}) d \mathbf{x}$ can be replaced by $\int_{\Omega} \nabla \mathbf{u}: \nabla \mathbf{v} d \mathbf{x}$ since $\mathbf{v}$ is divergence free and in $W_{0, p}$. Also the gravity $\mathbf{g}$ in (4.15) can be absorbed into the pressure term.

4.4. Space Approximation and Time-Discretization for the Fluid-Particle Interaction Sub-problem. The space approximation of problem (4.15)-(4.20) was performed via a finite element method. We have chosen $P_{1}-i s o-P_{2}$ and $P_{1}$ finite elements to approximate the velocity field and pressure, respectively (as in Bristeau et al. [3]). More precisely, if $h$ denotes a space discretization step, we introduce a finite element triangulation $\mathcal{T}_{h}$ of $\bar{\Omega}$ and a finite element triangulation $\mathcal{T}_{2 h}$ of $\bar{\Omega}$ where $\mathcal{T}_{2 h}$ is a triangulation which is twice coarser than $\mathcal{T}_{h}$. (In practice we construct $\mathcal{T}_{2 h}$ first and then $\mathcal{T}_{h}$ by joining the midpoints of the edges of $\mathcal{T}_{2 h}$, thereby dividing each triangle of $\mathcal{T}_{2 h}$ into 4 similar sub-triangles as shown in Figure 4.2.)

We then approximate function spaces $W_{\mathbf{g}_{0}, p}, W_{0, p}, L^{2}$ and $L_{0}^{2}$ by the following finite dimensional spaces respectively,

$$
\begin{gathered}
W_{\mathbf{g}_{0}, h}(t)=\left\{\mathbf{v}_{h}\left|\mathbf{v}_{h} \in\left(C^{0}(\bar{\Omega})\right)^{2}, \mathbf{v}_{h}\right|_{T} \in P_{1} \times P_{1}, \forall T \in \mathcal{T}_{h}, \mathbf{v}_{h}=\mathbf{g}_{0}^{h}(t) \text { on } \Gamma_{t},\right. \\
\left.\mathbf{v}_{h}=\mathbf{0} \text { on } \Gamma_{b}, \text { and } \mathbf{v}_{h} \text { is } x_{1} \text {-periodic }\right\} \\
W_{0, h}=\left\{\mathbf{v}_{h}\left|\mathbf{v}_{h} \in\left(C^{0}(\bar{\Omega})\right)^{2}, \mathbf{v}_{h}\right|_{T} \in P_{1} \times P_{1}, \forall T \in \mathcal{T}_{h}, \mathbf{v}_{h}=\mathbf{0} \text { on } \Gamma_{t} \cup \Gamma_{b},\right. \\
\text { and } \left.\mathbf{v}_{h} \text { is } x_{1} \text {-periodic }\right\} \\
L_{h}^{2}=\left\{q_{h}\left|q_{h} \in C^{0}(\bar{\Omega}), q_{h}\right|_{T} \in P_{1}, \forall T \in \mathcal{T}_{2 h}, q_{h} \text { is } x_{1} \text {-periodic }\right\} \\
L_{0, h}^{2}=\left\{q_{h} \mid q_{h} \in L_{h}^{2}, \int_{\Omega} q_{h} d \mathbf{x}=0\right\}
\end{gathered}
$$

where $\mathbf{g}_{0}^{h}$ is a discrete approximation of $\mathbf{g}_{0}$. Here $P_{1}$ is the space of polynomials in two variables of degree $\leq 1$.

To simplify notation, in the remaining part of this section we will be assuming the presence of only one particle $B(t)$ which is approximated by $B_{h}(t)$, and covered by a 


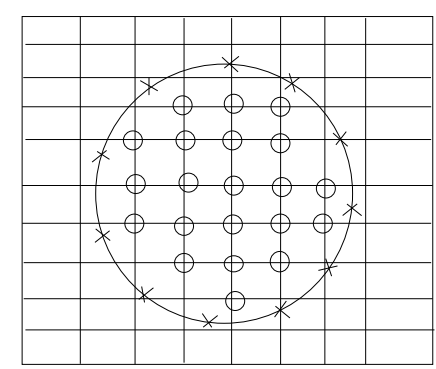

FIG. 4.3. An example of a set of collocation points chosen for enforcing the rigid body motion inside the disk and at its boundary.

set of grid points $\mathbf{x}_{i}, i=1, \ldots, N$. See Figure 4.3. The generalization of the algorithm to $J$ particles is performed in the spirit of the algorithm presented in the previous section.

A finite-dimensional approximation of the space of Lagrange multiplier(s) $\Lambda_{0}(t)$ is defined as follows. Let $\left\{\mathbf{x}_{i}\right\}_{i=1}^{N}$ be a set of points covering $\overline{B_{h}(t)}$. First define the space

$$
\Lambda_{h}(t)=\left\{\boldsymbol{\mu}_{h} \mid \boldsymbol{\mu}_{h}=\sum_{i=1}^{N} \boldsymbol{\mu}_{i} \delta\left(\mathbf{x}-\mathbf{x}_{i}\right), \boldsymbol{\mu}_{i} \in \mathbb{R}^{2}, \forall i=1, \ldots, N\right\}
$$

where $\delta(\cdot)$ is the Dirac measure at $\mathbf{x}=\mathbf{0}$. Then, instead of the scalar product of $\left(H^{1}\left(B_{h}(t)\right)\right)^{2}$, use $<\cdot, \cdot>_{B_{h}(t)}$ defined by

$$
<\boldsymbol{\mu}_{h}, \mathbf{v}_{h}>_{B_{h}(t)}=\sum_{i=1}^{N} \boldsymbol{\mu}_{i} \cdot \mathbf{v}_{h}\left(\mathbf{x}_{i}\right), \forall \boldsymbol{\mu}_{h} \in \Lambda_{h}(t), \mathbf{v}_{h} \in W_{0, h}
$$

As noted in [17], even though this is not consistent with (4.14), this approach is meaningful for the discrete problem since it amounts to forcing the rigid body motion of $B(t)$ via a collocation method. For more details, please see [17].

We now define an approximation of $\Lambda_{0}(t)$ by

$\Lambda_{0, h}(t)=\left\{\boldsymbol{\mu}_{h} \mid \boldsymbol{\mu}_{h} \in \Lambda_{h}(t),<\boldsymbol{\mu}_{h}, \mathbf{e}_{i}>_{B_{h}(t)}=0, i=1,2,<\boldsymbol{\mu}_{h},\left(\mathbf{x}-\mathbf{G}_{j}\right)^{\perp}>_{B_{h}(t)}=0\right\}$.

Using the above finite dimensional spaces leads to the following approximation of problem (4.15)-(4.20):

For a.a. $t>0$, find $\mathbf{u}(t) \in W_{\mathbf{g}_{0}, h}(t), p(t) \in L_{0, h}^{2}, \mathbf{V}_{\mathbf{G}}(t) \in \mathbb{R}^{2}, \mathbf{G}(t) \in \mathbb{R}^{2}$, $\omega(t) \in \mathbb{R}, \boldsymbol{\lambda}_{h}(t) \in \Lambda_{0, h}(t)$ such that 


$$
\begin{aligned}
& \left\{\begin{array}{l}
\rho_{f} \int_{\Omega}\left[\frac{\partial \mathbf{u}_{h}}{\partial t}+\left(\mathbf{u}_{h} \cdot \boldsymbol{\nabla}\right) \mathbf{u}_{h}\right] \cdot \mathbf{v} d \mathbf{x}+\mu_{f} \int_{\Omega} \boldsymbol{\nabla} \mathbf{u}_{h}: \nabla \mathbf{v} d \mathbf{x} \\
\quad-\int_{\Omega} p_{h} \boldsymbol{\nabla} \cdot \mathbf{v} d \mathbf{x}-<\boldsymbol{\lambda}_{h}, \mathbf{v}>_{B_{h}(t)}=\int_{\Omega} \mathbf{F} \cdot \mathbf{v} d \mathbf{x}, \forall \mathbf{v} \in W_{0, h},
\end{array}\right. \\
& \int_{\Omega} q \boldsymbol{\nabla} \cdot \mathbf{u}_{h}(t) d \mathbf{x}=0, \forall q \in L_{h}^{2}, \\
& <\boldsymbol{\mu}, \mathbf{u}_{h}(t)>_{B_{h}(t)}=0, \forall \boldsymbol{\mu} \in \Lambda_{0, h}(t), \\
& \frac{d \mathbf{G}}{d t}=\mathbf{V}_{\mathbf{G}} \\
& \mathbf{V}_{\mathbf{G}}(0)=\mathbf{V}_{\mathbf{G}}^{0}, \omega(0)=\omega^{0}, \mathbf{G}(0)=\mathbf{G}^{0}=\left\{G_{1}^{0}, G_{2}^{0}\right\}^{t}, \\
& \mathbf{u}_{h}(\mathbf{x}, 0)=\overline{\mathbf{u}}_{0, h}(\mathbf{x})\left(\text { with } \boldsymbol{\nabla} \cdot \overline{\mathbf{u}}_{0, h}=0\right) \text {. }
\end{aligned}
$$

Problem (4.25)-(4.30) is discretized in time by using the Lie's scheme and backward Euler scheme at some fractional steps in order to deal with the following subproblems separately:

1. The incompressibility condition and the related unknown pressure;

2. The advection term;

3. The diffusion term;

4. The rigid-body motion and the related Lagrange multiplier $\Lambda(t)$.

Although, this method is only first-order accurate, its low-order accuracy is compensated by good stability and robustness properties. (This scheme can be made second-order accurate by symmetrization [17].)

We now present this scheme coupled with the adhesion and repulsion force contributions giving rise to the numerical scheme for the solution of the fluid-particle interaction and adhesion problem.

4.5. The Numerical Scheme for the Fluid-Particle Interaction and Adhesion Problem. By employing the time-splitting approach described in Section 4.2, and by employing the time and space discretization of the fluid-particle interaction sub-problem described in Section 4.4, we obtain the following numerical scheme for the fluid-particle interaction and adhesion problem:

$$
\mathbf{u}^{0}=\overline{\mathbf{u}}_{0, h}, \mathbf{V}_{\mathbf{G}}^{0}, \omega^{0} \text {, and } \mathbf{G}^{0} \text { are given }
$$

For $n \geq 0$, knowing $\mathbf{u}^{n}, \mathbf{V}_{\mathbf{G}}^{n}$, $\omega^{n}$ and $\mathbf{G}^{n}$, compute $\mathbf{u}^{n+1 / 6}$ and $p^{n+1 / 6}$ via the solution of

$$
\left\{\begin{array}{l}
\rho_{f} \int_{\Omega} \frac{\mathbf{u}^{n+1 / 6}-\mathbf{u}^{n}}{\triangle t} \cdot \mathbf{v} d \mathbf{x}-\int_{\Omega} p^{n+1 / 6} \nabla \cdot \mathbf{v} d \mathbf{x}=0, \forall \mathbf{v} \in W_{0, h}, \\
\int_{\Omega} q \nabla \cdot \mathbf{u}^{n+1 / 6} d \mathbf{x}=0, \forall q \in L_{h}^{2} ; \mathbf{u}^{n+1 / 6} \in W_{\mathbf{g}_{0}, h}^{n+1}, p^{n+1 / 6} \in L_{0, h}^{2}
\end{array}\right.
$$

Then compute $\mathbf{u}^{n+2 / 6}$ via the solution of

$$
\begin{aligned}
& \left\{\begin{array}{l}
\int_{\Omega} \frac{\partial \mathbf{u}}{\partial t} \cdot \mathbf{v} d \mathbf{x}+\int_{\Omega}\left(\mathbf{u}^{n+1 / 6} \cdot \nabla\right) \mathbf{u} \cdot \mathbf{v} d \mathbf{x}=0, \forall \mathbf{v} \in W_{0, h}, \text { on }\left(t^{n}, t^{n+1}\right), \\
\mathbf{u}\left(t^{n}\right)=\mathbf{u}^{n+1 / 6} ; \mathbf{u}(t) \in W_{\mathbf{g}_{0}, h}^{n+1},
\end{array}\right. \\
& \mathbf{u}^{n+2 / 6}=\mathbf{u}\left(t^{n+1}\right) .
\end{aligned}
$$


Next, compute $\mathbf{u}^{n+3 / 6}$ via the solution of

$$
\left\{\begin{array}{l}
\rho_{f} \int_{\Omega} \frac{\mathbf{u}^{n+3 / 6}-\mathbf{u}^{n+2 / 6}}{\Delta t} \cdot \mathbf{v} d \mathbf{x}+\alpha \mu_{f} \int_{\Omega} \nabla \mathbf{u}^{n+3 / 6} \cdot \nabla \mathbf{v} d \mathbf{x}=0 \\
\forall \mathbf{v} \in W_{0, h} ; \quad \mathbf{u}^{n+3 / 6} \in W_{\mathbf{g}_{0}, h}^{n+1}
\end{array}\right.
$$

Now predict the position and the translation velocity of the center of mass of the particles as follows:

Take $\mathbf{V}_{\mathbf{G}}^{n+\frac{4}{6}, 0}=\mathbf{V}_{\mathbf{G}}^{n}$ and $\mathbf{G}^{n+\frac{4}{6}, 0}=\mathbf{G}^{n}$; then predict the new position of the particle via the following predictor-corrector technique:

For $k=1, \ldots, N$,

Calculate $\mathbf{F}^{a}$ via Cell Dynamic Adhesion Algorithm,

Calculate $\mathbf{F}^{a r}=\mathbf{F}^{a}+\mathbf{F}^{r}$,

$\widehat{\mathbf{V}}_{\mathbf{G}}^{n+\frac{4}{6}, k}=\mathbf{V}_{\mathbf{G}}^{n+\frac{4}{6}, k-1}+M^{-1} \mathbf{F}^{a r}\left(\mathbf{G}^{n+\frac{4}{6}, k-1}\right) \triangle t / 2 N$,

$\widehat{\mathbf{G}}^{n+\frac{4}{6}, k}=\mathbf{G}^{n+\frac{4}{6}, k-1}+\left(\widehat{\mathbf{V}}_{\mathbf{G}}^{n+\frac{4}{6}, k}+\mathbf{V}_{\mathbf{G}}^{n+\frac{4}{6}, k-1}\right) \triangle t / 4 N$,

$\mathbf{V}_{\mathbf{G}}^{n+\frac{4}{6}, k}=\mathbf{V}_{\mathbf{G}}^{n+\frac{4}{6}, k-1}+M^{-1}\left(\mathbf{F}^{a r}\left(\widehat{\mathbf{G}}^{n+\frac{4}{6}, k}\right)+\mathbf{F}^{a r}\left(\mathbf{G}^{n+\frac{4}{6}, k-1}\right)\right) \Delta t / 4 N$,

$\mathbf{G}^{n+\frac{4}{6}, k}=\mathbf{G}^{n+\frac{4}{6}, k-1}+\left(\mathbf{V}_{\mathbf{G}}^{n+\frac{4}{6}, k}+\mathbf{V}_{\mathbf{G}}^{n+\frac{4}{6}, k-1}\right) \triangle t / 4 N$,

enddo;

and let $\mathbf{V}_{\mathbf{G}}^{n+\frac{4}{6}}=\mathbf{V}_{\mathbf{G}}^{n+\frac{4}{6}, N}, \mathbf{G}^{n+\frac{4}{6}}=\mathbf{G}^{n+\frac{4}{6}, N}$.

Now, compute $\mathbf{u}^{n+5 / 6}, \lambda^{n+5 / 6}, \mathbf{V}_{\mathbf{G}}^{n+5 / 6}$, and $\omega^{n+5 / 6}$ via the solution of

$$
\left\{\begin{array}{l}
\rho_{f} \int_{\Omega} \frac{\mathbf{u}^{n+5 / 6}-\mathbf{u}^{n+3 / 6}}{\triangle t} \cdot \mathbf{v} d \mathbf{x}+\beta \mu_{f} \int_{\Omega} \nabla \mathbf{u}^{n+5 / 6} \cdot \nabla \mathbf{v} d \mathbf{x} \\
=<\boldsymbol{\lambda}, \mathbf{v}>_{B_{h}^{n+4 / 6}}, \forall \mathbf{v} \in W_{0, h}, \\
<\boldsymbol{\mu}, \mathbf{u}^{n+5 / 6}>_{B_{h}^{n+4 / 6}}=0, \forall \boldsymbol{\mu} \in \Lambda_{0, h}^{n+4 / 6} ; \mathbf{u}^{n+5 / 6} \in W_{\mathbf{g}_{0}, h}^{n+1}, \boldsymbol{\lambda}^{n+5 / 6} \in \Lambda_{0, h}^{n+4 / 6},
\end{array}\right.
$$

and solve for $\mathbf{V}_{\mathbf{G}}^{n+5 / 6}$ and $\omega^{n+5 / 6}$ from

$$
\left\{\begin{array}{l}
<\mathbf{e}_{i}, \mathbf{u}^{n+5 / 6}-\mathbf{V}_{\mathbf{G}}^{n+5 / 6}-\omega^{n+5 / 6} \overrightarrow{G^{n+4 / 6} x}>_{B_{h}^{n+4 / 6}}=0, \text { for } i=1,2, \\
<{\overrightarrow{G^{n+4 / 6} x}}^{\perp}, \mathbf{u}^{n+5 / 6}-\mathbf{V}_{\mathbf{G}}^{n+5 / 6}-\omega^{n+5 / 6} \overrightarrow{G^{n+4 / 6} x}>_{B_{h}^{n+4 / 6}}=0,
\end{array}\right.
$$

Finally, take $\mathbf{V}_{\mathbf{G}}^{n+1,0}=\mathbf{V}_{\mathbf{G}}^{n+5 / 6}$ and $\mathbf{G}^{n+1,0}=\mathbf{G}^{n+4 / 6}$; then predict the final position and translation velocity as follows: 
For $k=1, \ldots, N$,

Calculate $\mathbf{F}^{a}$ via Cell Dynamic Adhesion Algorithm,

Calculate $\mathbf{F}^{a r}=\mathbf{F}^{a}+\mathbf{F}^{r}$,

$\widehat{\mathbf{V}}_{\mathbf{G}}^{n+1, k}=\mathbf{V}_{\mathbf{G}}^{n+1, k-1}+M^{-1} \mathbf{F}^{a r}\left(\mathbf{G}^{n+1, k-1}\right) \triangle t / 2 N$,

$\widehat{\mathbf{G}}^{n+1, k}=\mathbf{G}^{n+1, k-1}+\left(\widehat{\mathbf{V}}_{\mathbf{G}}^{n+1, k}+\mathbf{V}_{\mathbf{G}}^{n+1, k-1}\right) \triangle t / 4 N$,

$\mathbf{V}_{\mathbf{G}}^{n+1, k}=\mathbf{V}_{\mathbf{G}}^{n+1, k-1}+M^{-1}\left(\mathbf{F}^{a r}\left(\widehat{\mathbf{G}}^{n+1, k}\right)+\mathbf{F}^{a r}\left(\mathbf{G}^{n+1, k-1}\right)\right) \triangle t / 4 N$,

$\mathbf{G}^{n+1, k}=\mathbf{G}^{n+1, k-1}+\left(\mathbf{V}_{\mathbf{G}}^{n+1, k}+\mathbf{V}_{\mathbf{G}}^{n+1, k-1}\right) \triangle t / 4 N$,

enddo;

Complete the final step by setting $\mathbf{V}_{\mathbf{G}}^{n+1}=\mathbf{V}_{\mathbf{G}}^{n+1, N}, \mathbf{G}^{n+1}=\mathbf{G}^{n+1, N}$; and $\mathbf{u}^{n+1}=$ $\mathbf{u}^{n+5 / 6}, \omega^{n+1}=\omega^{n+5 / 6}$.

In the above algorithm (4.31)-(4.48), we have $t^{n+s}=(n+s) \triangle t, W_{\mathbf{g}_{0}, h}^{n+1}=W_{\mathbf{g}_{0}, h}\left(t^{n+1}\right)$, $\Lambda_{0, h}^{n+s}=\Lambda_{0, h}\left(t^{n+s}\right), B_{h}^{n+s}$ is the region occupied by the particle centered at $\mathbf{G}^{n+s}$, and $\alpha$ and $\beta$ verify $\alpha+\beta=1$; we have chosen $\alpha=1$ and $\beta=0$ in the numerical simulations discussed in the next section.

The degenerated quasi-Stokes problem (4.32) is solved by a preconditioned conjugate gradient method introduced in [18], in which discrete elliptic problems from the preconditioning are solved by a matrix-free fast solver from FISHPAK by Adams et al. [1]. The advection problem (4.33) for the velocity field is solved by a wave-like method as in [11]. Problem (4.35) is a classical discrete elliptic problem which can be solved by the same matrix-free fast solver. To enforce the rigid body motion inside the region occupied by the particles, we have applied the conjugate gradient method discussed in $[29,30]$.
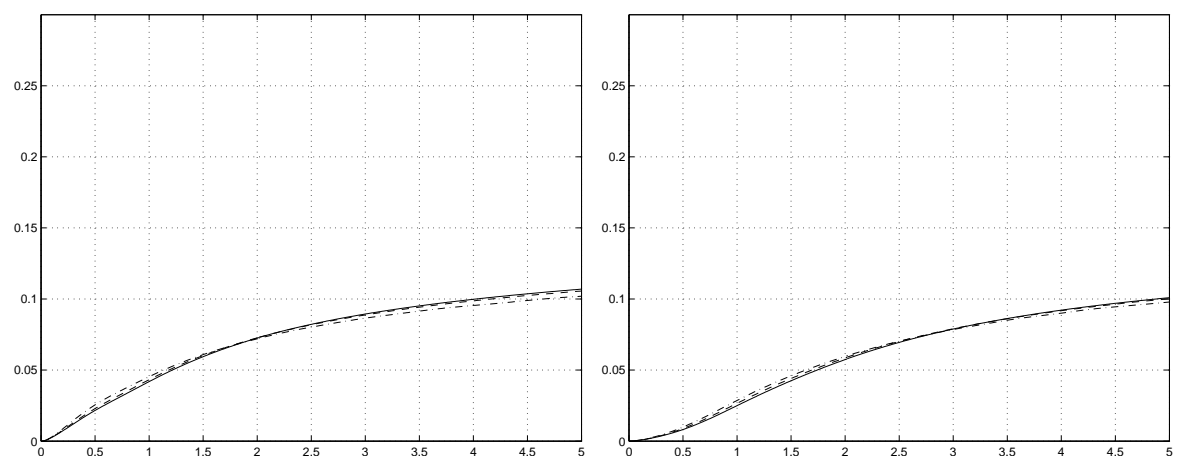

FIG. 4.4. The figures show the histories of the height of the center of mass of a circular disk in shear flow (left) and of an elliptical particle in shear flow (right) obtained with different mesh sizes: $h=1 / 128$ (dash-dot line), 1/196 (dashed line) and 1/256 (solid line).

It is important to notice that in our approach, we do not need to compute the lift and drag forces via the integration on the boundary of a cell. We obtain the cell translation velocity and angular velocity by a direct calculation when enforcing the rigid body motion in the region occupied by the cell. Therefore, computations with different mesh sizes produce consistent hydrodynamic forces applied to the cells. To strenghten this point, we show in Figure 4.4 the histories of the height of the 
center of mass of a circular disk in shear flow (left) and of an elliptical particle in shear flow (right) obtained with different mesh sizes: $\mathrm{h}=1 / 128$ (dash-dot line), 1/196 (dashed line) and 1/256 (solid line). These figures show that the computational results are consistent when reducing the mesh size, thereby showing consistent lift force for particles in shear flow. The results shown in Figure 4.4 were obtained with the time step $\Delta t=10^{-3}$, the semi-major axis $s_{1}=0.125$ and the semi-minor axis $s_{2}=0.0625$. The domain size is 1 unit times 1 unit in space and the shear rate is 10 . The viscosity of fluid is 0.012. Adhesion forces were not included in these simulations.

The fluid-particle interaction algorithm for neutrally buoyant particles was validated in [29].

5. Numerical Results and Discussion. In this section we present numerical results that show how the percentage of detached cells in shear flow depends on the magnitude of shear flow, the type of shear flow (linear versus oscillatory), the number of adhesion molecules covering each cell, and the initial distribution and number of adhered cells at the bottom surface $\Gamma_{b}$.

We consider 20 cells immersed in the fluid. The $2 \mathrm{D}$ computational domain is $\Omega=(0,370) \times(0,50) \mu m^{2}$. The cells have the shape of an ellipse, see Figure 5.1, with the long semi-axis $r_{a}$ equal to $5 \mu \mathrm{m}$ and the short semi-axis $r_{b}$ equal to $4 \mu \mathrm{m}$, as was observed in experiments [34].

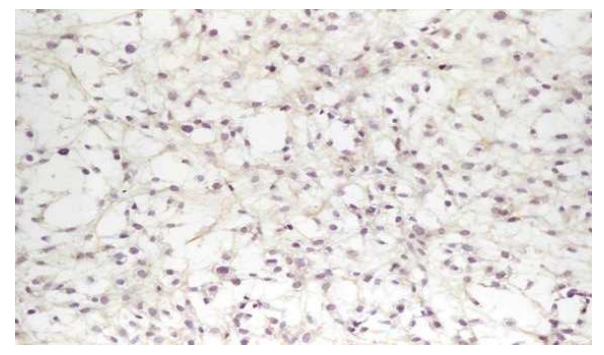

FIG. 5.1. Immunocythochemistry of passaged chondrocytes in a cell culture medium supplemented with vitamin $C$ for improved collagen synthesis (Scott-Burden, Rosenstrauch et al. [34].) Positive staining for collagen II indicates the presence of chondrocytes (dark elliptical dots).

The mesh size $h$ for the flow field is $0.3125 \mu m$, and the time step $\triangle t$ is $4 \times 10^{-6}$ s. The parameters used in the simulations are given in Table 1.

As stated in Section 4.1, the velocity boundary conditions at the boundary of $\Omega$ are as follows: a given velocity $\mathbf{g}_{0}(t)$ on the top boundary $\Gamma_{t}$, zero velocity at the bottom boundary $\Gamma_{b}$, and periodic boundary conditions at $\Gamma_{\text {in }}$ and $\Gamma_{\text {out }}$. We consider two types of shear flow: linear shear flow and oscillatory flow. In the case of oscillatory flow, a given velocity at the top boundary was taken to be of the following form

$$
\mathbf{g}_{0}(t)=(a \sin (\omega t), 0), \text { with frequency } 1 H z \text {. }
$$

Each cell is covered with adhesion molecules represented by 500 receptors randomly distributed over the cell surface. The initial value of the spring constant $\sigma$ for each adhesion molecule was chosen randomly between 1 and 5 and was then multiplied by $10^{-3}$ dyne/cm, and the corresponding transition state spring constant $\sigma_{t s}$ was then assigned to be 1.1 times the spring constant $\sigma$ ("catch bonds" [12]). After the exposure to shear flow, the spring constants increased over time as explained in the Modified Dynamic Adhesion Algorithm presented in Section 3.2. 
The fluid and the cells were at rest initially. The cells were placed in the contact region with the bottom surface, and were left there until the number of established bonds reached a steady state, which took about $0.2 \mathrm{~s}$. At $0.2 \mathrm{~s}$ shear flow was applied.

\begin{tabular}{llll}
\hline Parameters & Definition & range & reference \\
\hline$r$ & cell radius & $4.0-5.0 \mu \mathrm{m}$ & {$[9,23]$} \\
$N_{r}$ & receptor number/cell & $500-700$ & {$[25,31]$} \\
$\lambda$ & equilibrium bond length & $0.25 \mu \mathrm{m}$ & {$[31]$} \\
$\sigma$ & spring constant & $10^{-3}-0.05 \mathrm{dyne} / \mathrm{cm}$ & {$[31]$} \\
$\sigma_{t s}$ & transition spring constant & $0.0011-0.055 \mathrm{dyne} / \mathrm{cm}$ & {$[20]$} \\
$\mu_{f}$ & viscosity & $0.01 \mathrm{~g} / \mathrm{cm} \cdot \mathrm{s}$ & {$[7]$} \\
$\rho_{f}$ & fluid density & $1.0 \mathrm{~g} / \mathrm{cm}^{3}$ & {$[23]$} \\
$\dot{\gamma}$ & shear rate & $50-400 \mathrm{~s}^{-1}$ & {$[20]$} \\
$H_{c}$ & cut-off length & $0.5 \mu \mathrm{m}$ & {$[7,31]$} \\
$T$ & temperature & $310 \mathrm{~K}$ & {$[6]$} \\
$k_{f}^{0}$ & forward reaction rate & $500.0 \mathrm{~s}^{-1}$ & {$[23]$} \\
$k_{r}^{0}$ & reverse reaction rate & $50.0 \mathrm{~s}^{-1}$ & {$[7,25]$} \\
\hline
\end{tabular}

Table 1. Simulation Parameters.

5.1. Magnitude of Linear Shear Flow and Detachment Percentage. We first studied the influence of the magnitude of linear shear flow on the detachment percentage. Figure 5.2 shows the detachment percentages evolving over time in shear flow at shear rates $\dot{\gamma}=50,70$, and $80 \mathrm{~s}^{-1}$ respectively. At shear rate $\dot{\gamma}=50 \mathrm{~s}^{-1}$, the percentage of detached cells increased from $0 \%$ to $10 \%$ from $\mathrm{t}=0.2 \mathrm{~s}$ to $\mathrm{t}=0.362 \mathrm{~s}$; after $\mathrm{t}=0.362 \mathrm{~s}$ the detachment percentage became constant. At shear rates $\dot{\gamma}=70$ and $80 \mathrm{~s}^{-1}$, the detachment percentages increased from $0 \%$ to $35 \%$ and $0 \%$ to $60 \%$ until $\mathrm{t}=0.766 \mathrm{~s}$ and $\mathrm{t}=0.796 \mathrm{~s}$ respectively. After $t=0.766$ the detachment percentage leveled off. Figure 5.2 indicates that in linear shear flow the detachment percentage increases over time during the early stages of detachment; the detachment process then slows down and it eventually stops once a strong adhesion is achieved. This qualitative behavior of cummulative cell detachment as a function of time corresponds to the experimentally observed cell detachment reported in [34].

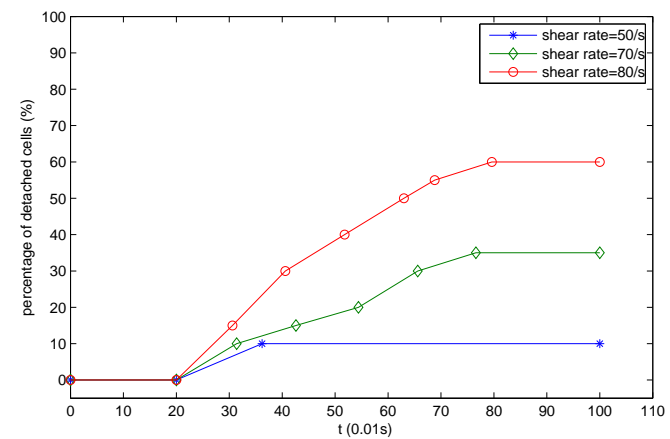

FIG. 5.2. Detachment percentage over time in linear shear flow with 20 cells.

Figure 5.3 shows the snapshots of positions of 20 cells in shear flow with the shear 
rate of $70 \mathrm{~s}^{-1}$ at $t=0,0.362,0.544,0.766$, and $0.958 \mathrm{~s}$. The snapshots quite clearly depict the process of cell detachment from the surface. Figure 5.3 (a) shows the initial position of 20 cells which were in the contact region with the bottom surface. The linear shear flow was applied at $\mathrm{t}=0.2 \mathrm{~s}$ (not shown here). At $\mathrm{t}=0.362 \mathrm{~s}$ and $0.544 \mathrm{~s}$, the percentage of detached cells was $10 \%$ and $20 \%$ respectively. Notice how cells roll before they detach. Also notice that due to the periodic inlet and outlet boundary conditions, the detached cells that left the domain after the snapshot in picture (b) was taken, appear near the inlet of the domain in the snapshot (c). After $t=0.766 \mathrm{~s}$, the detachment rate slowed down, and the cumulative percentage of detached cells did no exceed $35 \%$ at $\mathrm{t}=0.958$. In Figure 5.4 we show the velocity and pressure gradient
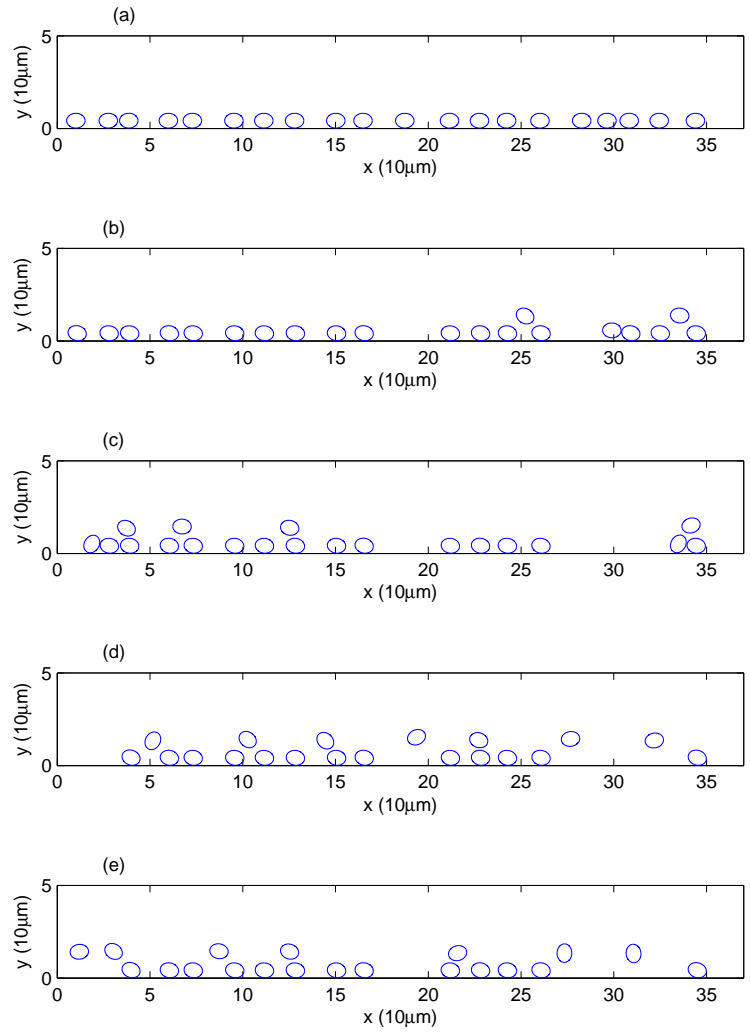

Fig. 5.3. Snapshots of 20 cells in a shear flow at $t=0.00 \mathrm{~s}(a), 0.362 \mathrm{~s}(\mathrm{~b}), 0.544 \mathrm{~s}(\mathrm{c}), 0.766 \mathrm{~s}$ (d), and $0.958 \mathrm{~s}(\mathrm{e})$ (shear rate $\left.=70 \mathrm{~s}^{-1}\right)$. The percentage of detached cells was $35 \%$ at $t=0.958 \mathrm{~s}$.

distribution at $\mathrm{t}=0.406 \mathrm{~s}$. It is interesting to notice the small velocity deviation from the linear shear distribution in the top figure, as well as the pressure deviations around the particles shown at the bottom figure.

Based on the detachment results presented above, we reach the following conclusions: (1) the higher the linear shear rate, the larger the percentage of detached cells, and (2) the percentage of detached cells levels off after a certain time. This behavior has been observed in experiments with auricular chondrocytes, reported in [34], and in experiments with articular chondrocytes exposed to fluid flow-induced shear stress, reported in [33]. We notice, however, that the magnitude of shear stress applied to articular chondrocytes, reported in [33], was much higher than the one used in our 

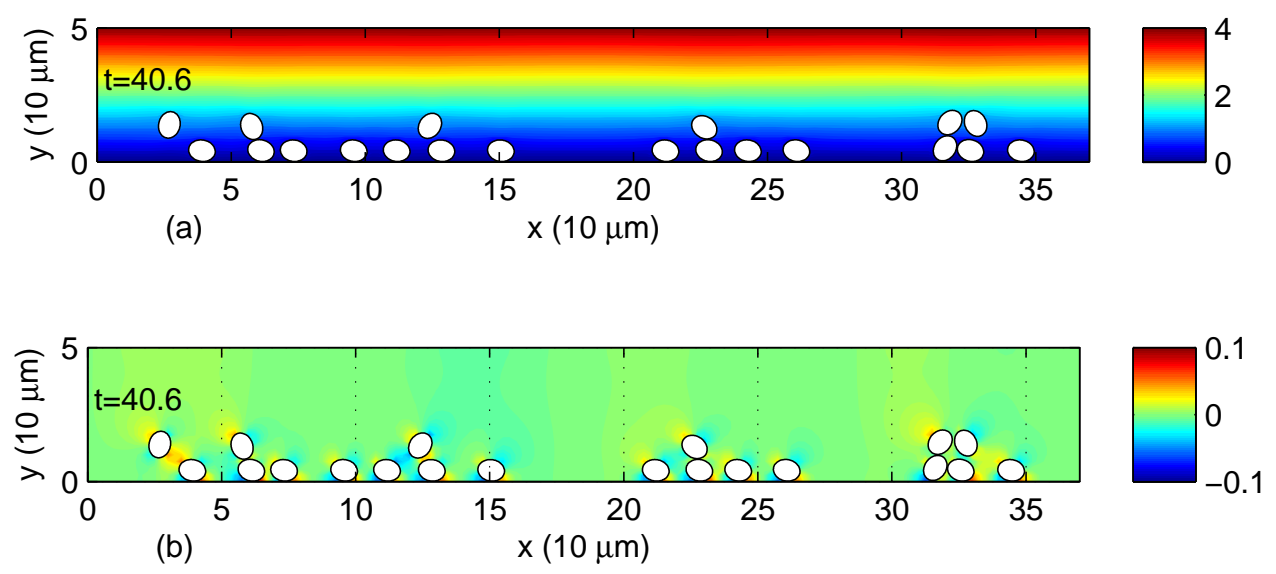

FIG. 5.4. The figure shows a snapshot of 20 cells in shear flow (shear rate $=80 \mathrm{~s}^{-1}$ ) at $t=0.406 \mathrm{~s}$. The top figure shows the velocity distribution in $\mu \mathrm{m} / \mathrm{s}$ and the figure at the bottom show the pressure gradient distribution in Pascals.

numerical investigation and that the time-scale at which chondrocyte detachment was observed was longer (one minute). It is natural to expect longer cell detachment time interval in experiments reported in [33] since, among other things, the duration of the initial cell seeding in [33] was longer than that considered in the present manuscript.

5.2. Linear Shear Flow versus Oscillatory Flow. Here we investigate the influence of oscillatory flow on cell detachment. The cells were exposed to the flow generated by the boundary data at the top boundary given by $\mathbf{g}_{0}(t)=(a \sin (\omega t), 0)$, with frequency $1 \mathrm{~Hz}$, and with the maximum shear rate ranging from 10 to $80 \mathrm{~s}^{-1}$. The total percentage of detached cells was then compared with that obtained with the linear shear rate. The calculated detachment percentages at $\mathrm{t}=1.00 \mathrm{~s}$ are summarized in Table 2.

\begin{tabular}{ccc}
\hline Shear rate $\left(\mathrm{s}^{-1}\right)$ & Detachment \% (Linear) & Detachment \% (Oscillatory) \\
\hline 10 & 0 & 0 \\
50 & 10 & 10 \\
70 & 35 & 20 \\
80 & 60 & 35 \\
\hline
\end{tabular}

Table 2. The calculated total detachment percentages for 20 cells at $t=1.00 \mathrm{~s}$ with linear shear flow (middle) and oscillatory flow (right).

From Table 2, we can see that no cells were detached from the surface in either flow when the shear rate was $10 \mathrm{~s}^{-1}$ while the detachment percentage increased from $10 \%$ to $60 \%$ in shear flow and from $10 \%$ to $35 \%$ in oscillatory flow, respectively, when the shear rate increased from $50 \mathrm{~s}^{-1}$ to $80 \mathrm{~s}^{-1}$. Figure 5.5 shows the effect of shear rate in the two flows on cell detachment. We remark that in both flows the percentage of detached cells as a function of time becomes constant after a certain time. See Figure 5.6 for the dynamics of cell detachment in oscillatory flow. 


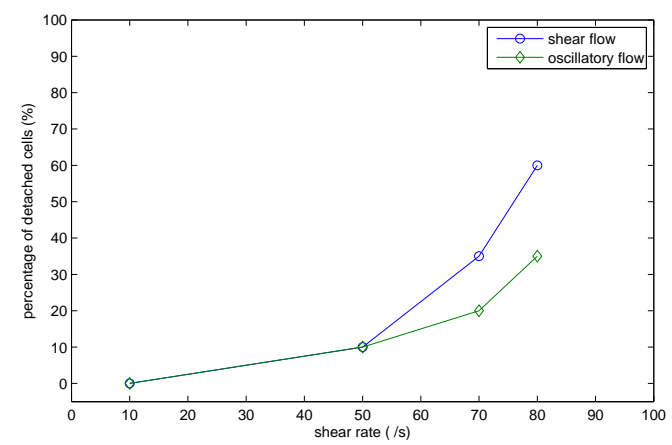

FIG. 5.5. The effect of oscillatory flow versus linear shear flow on cell detachment of 20 cells.

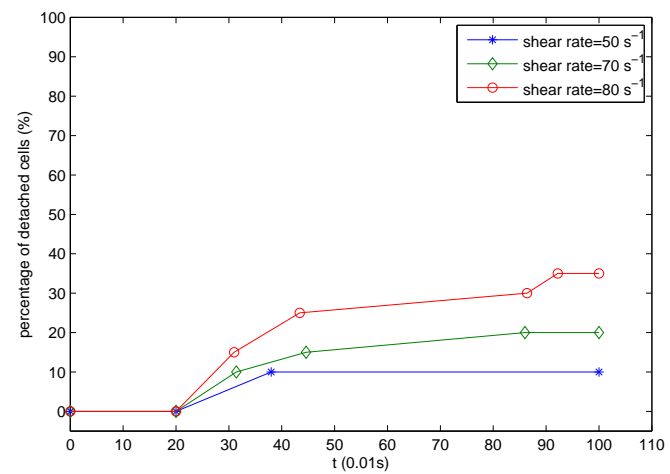

FIG. 5.6. The dynamics of cell detachment in oscillatory flow.

Based on these results we conclude that oscillatory flow produces better results in the sense that the total number of detached cells in oscillatory shear flow was less than that in linear shear flow. This is, however, a direct consequence of the fact that oscillatory shear flow produces less shear stress on the cells at the bottom of the channel and increases the residence time of particles near the substrate. In order to truly model the experimental observations which indicate that cyclic loading of articular chondrocytes accelerates the formation of tissue-engineered cartilage (see, e.g., [38]), our model would have to incorporate a sophisticated dependence of the bond strength on the magnitude and direction of shear stress, which is not included in the present paper, and is a subject of our current and future research.

5.3. Initial Distribution of Cells and Cell Detachment. We now investigate the effect of the initial distribution of cells on cell detachment percentage. More precisely, we are interested in comparing the results on cell detachment obtained with the randomly distributed cells (which were used in all the simulations presented in this manuscript) with those obtained with the cells that are uniformly distributed, where the initial separation of cells is the average of the cell separation obtained in random initial distribution. Thus, we ran simulations with the following two sets of initial cell distributions:

- random initial cell distribution (where separation varied from $0.625 \mu m$ to 


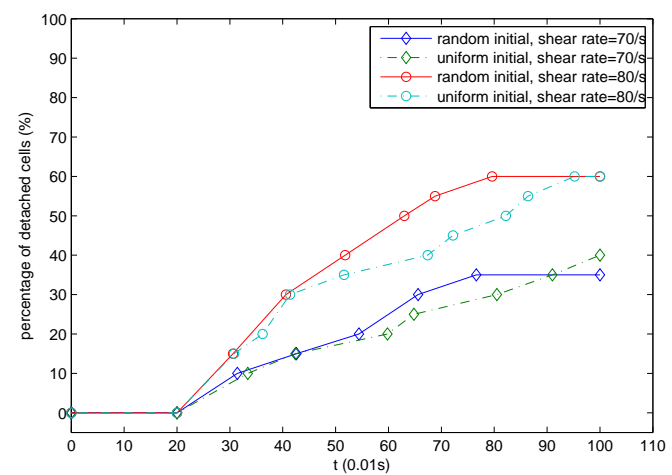

FIG. 5.7. The effect of initial separation of 20 cells on detachment percentage.

$15.625 \mu \mathrm{m})$, and

- uniform initial cell distribution (with the separation distance equal to $8.125 \mu \mathrm{m}$ ).

Graphs showing the detachment percentages over time with two different initial positions for two different shear rates are given in Figure 5.7. From Figure 5.7 we can see that the dynamics of cell detachment is different for the two distinct initial cell distributions, however, the difference is not large. In fact, the two curves corresponding to the same shear rate differ by only $5 \%$ at time $t=1$.

From these simulations we conclude that initial separation of cells influences the dynamics of cell detachment, but we expect that it will not influence the total detachment percentage in a significant way.

5.4. Detachment of $\mathbf{5 0}$ Cells. In addition to the tests presented above, we ran several simulations involving 50 cells initially seeded on the bottom surface. The 50 cells were distributed randomly over the bottom surface, however, the density of distribution was slightly higher than that of 20 cells. See Figure 5.8. We emphasize that, due to the computational intensity of the simulations, we have not seen anywhere in the literature simulations that involved more than 14 cells in a fluid-particle interaction and adhesion algorithms. Thus, these simulations provide new information regarding the adhesion and detachment of a larger number of cells to an artificial surface exposed to fluid-flow induced shear stress.

The computational domain we took in this simulation was $\Omega=(0,800) \times(0,50) \mu m^{2}$. The initial separation of cells was randomly assigned to range between $0.625 \mu \mathrm{m}$ to $10.625 \mu \mathrm{m}$. Everything else was kept the same as for the case of 20 cells. We performed the simulations with the shear rates of $10,50,60$, and $70 \mathrm{~s}^{-1}$ for the physical time duration of $1 \mathrm{~s}$. Table 3 shows the total detachment percentages for the different shear rates. We see that the detachment percentage for 50 cells does not differ in a significant way from the detachment percentage obtained with 20 cells that are slightly more sparsley distributed.

\begin{tabular}{cc}
\hline Shear rate $\left(\mathrm{s}^{-1}\right)$ & Cell detachment for 50 cells $(\%)$ \\
\hline 10 & 0 \\
50 & 4 \\
60 & 10 \\
70 & 34 \\
\hline
\end{tabular}




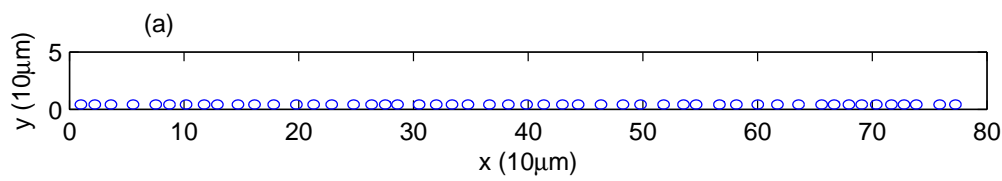

(b)
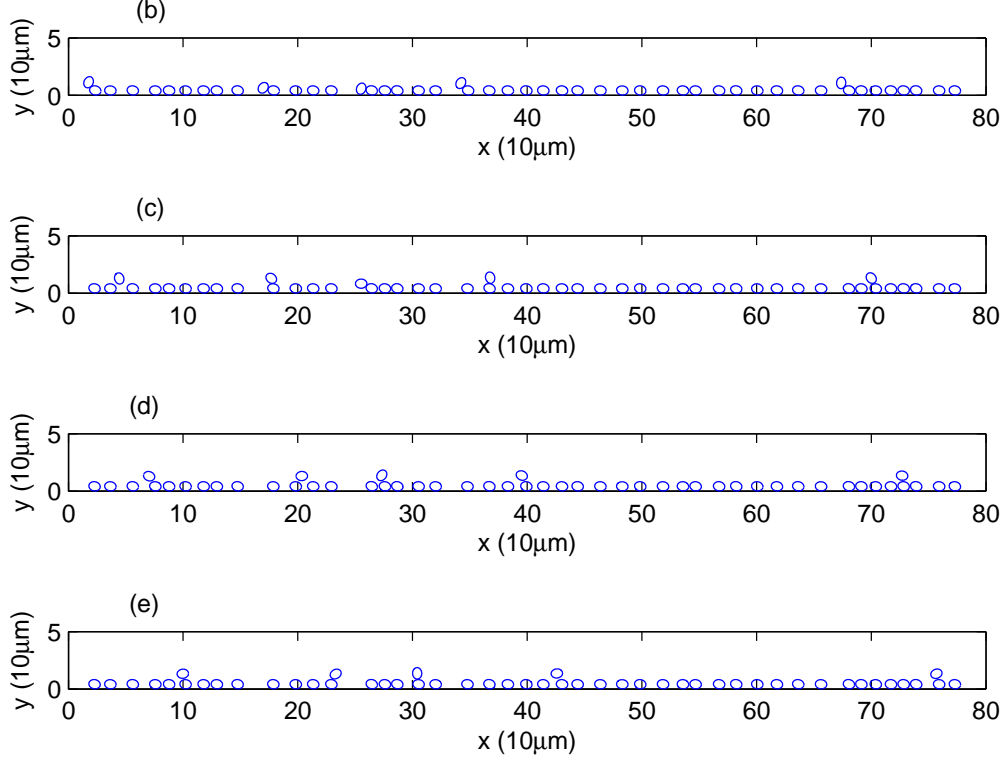

Fig. 5.8. Snapshots of 50 cells exposed to linear shear flow at $t=0.00 \mathrm{~s}(\mathrm{a}), 0.320 \mathrm{~s}(\mathrm{~b}), 0.360$ $s(c), 0.398 s(d)$, and $0.440 s(e)$ (shear rate $=60 \mathrm{~s}^{-1}$ ).

Table 3. The calculated total detachment percentages for $\mathbf{5 0}$ cells at $t=1.00 \mathrm{~s}$.

Figure 5.8 shows detachment of 50 cells at shear rate of $60 \mathrm{~s}^{-1}$, taken at 5 snapshots corresponding to $\mathrm{t}=0.00 \mathrm{~s}, \mathrm{t}=0.320 \mathrm{~s}, \mathrm{t}=0.360 \mathrm{~s}, \mathrm{t}=0.398 \mathrm{~s}$, and $\mathrm{t}=0.440 \mathrm{~s}$. After $\mathrm{t}=0.380 \mathrm{~s}$ the detachment rate reached a steady state. The total percentage of detached cells was $10 \%$.

Figure 5.9 shows the dynamics of cell detachment for 50 cells at the shear rate of $70 \mathrm{~s}^{-1}$ compared with the dynamics of cell detachment for 20 cells at the same shear rate. These results indicate that the detachment percentage does not depend in a substantial way on the total number of cells considered as long as they have roughly the same initial seeding density.

5.5. The Average Number and Strength of Bonds in Adhered Cells. In this section we "zoom" into the nano-scale phenomena to reveal the dynamics of bond association for adhered cells.

First, we followed the change in the number of bonds of adhered cells as a function of time at the shear rate of $70 s^{-1}$ for 20 cells. Each cell was covered with 500 adhesion molecules. Figure 5.10 shows the average number of bonds over time. One can see that the average number of bonds rapidly increased from 0 to about 40 by $\mathrm{t}=0.04 \mathrm{~s}$, and then fluctuated around 40 when the cells were at equilibrium. At $\mathrm{t}=0.2 \mathrm{~s}$, the shear flow was applied. No fluctuations in the number of bonds after the application of linear shear flow was recorded. The average number of bonds decreased slightly and 


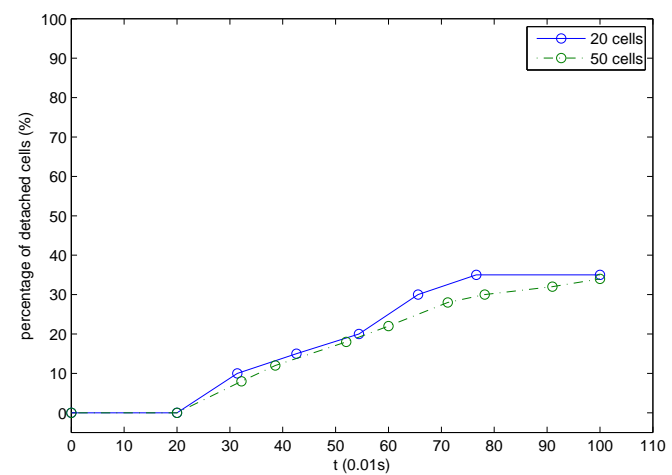

Fig. 5.9. A comparison of cell detachment dynamics for 50 cells versus 20 cells at the shear rate of $70 \mathrm{~s}^{-1}$.

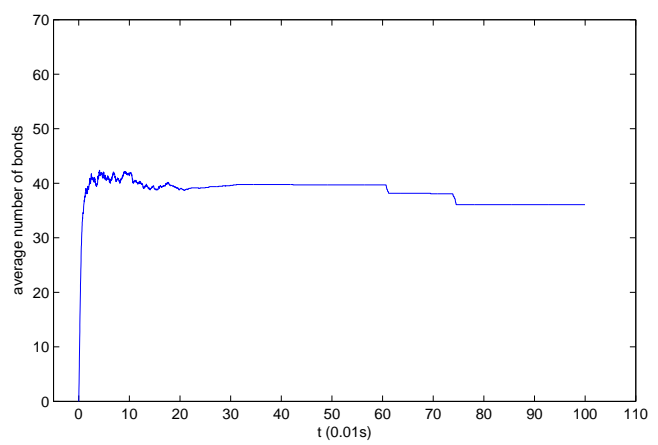

FIG. 5.10. The average number of bonds evolving over time for the adherent cells at shear rate $\dot{\gamma}=70 \mathrm{~s}^{-1}$.

appeared to have stayed constant after $\mathrm{t}=0.8 \mathrm{~s}$. The graph in Figure 5.10 should be viewed together with the graph in Figure 5.2 which shows the dynamics of cell detachment over time for shear rate of $70 \mathrm{~s}^{-1}$. One can notice that after $\mathrm{t}=0.8 \mathrm{~s}$ no additional cells were detached from the bottom surface and that the number of bonds after $0.8 \mathrm{~s}$ became constant. This indicates the formation of stable adhesion.

Another interesting information is the average strength of the spring constant of adhered cells in shear flow for different shear rates. Figure 5.11 shows the average strength for the spring constants at $t=1.0 \mathrm{~s}$ as a function of shear rate for the adherent cells. One can see that the average strength increased with shear rate. The values of the average spring constant were $3.00,3.21,3.49$, and 3.61 times $10^{-3}$ dyne $/ \mathrm{cm}$ when the shear rate was $10,50,70$, and $80 \mathrm{~s}^{-1}$ respectively. This indicates that adhered cells exposed to the higher shear rates embody stronger adhesion and thus a stronger tissue coating. This is to be expected since cells having low average spring constant values are swept away, leaving only those with higher values.

We make the following two conclusions: (1) Our algorithm indicates that longer exposure to shear-stress implies convergence to a steady state in the dynamic bond association and disassociation process, indicating the formation of a stable coating; (2) Stronger tissue coating will result with exposure to higher shear stress since, among 


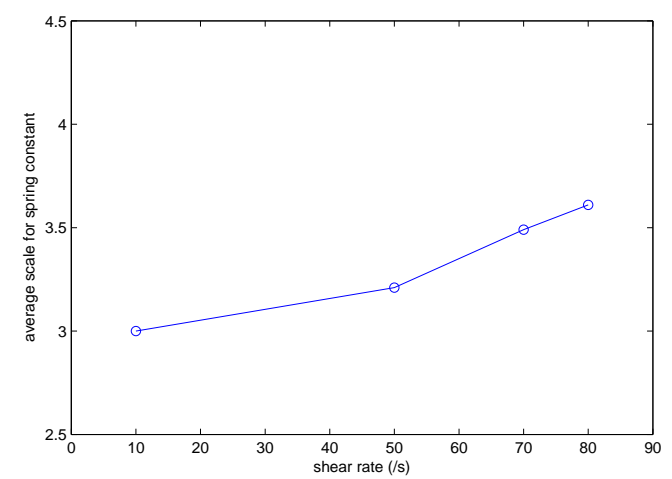

FIG. 5.11. The average spring constant (times $10^{-3}$ dyne $/ \mathrm{cm}$ ) versus shear rate. The graph shows data for the adherent cells.
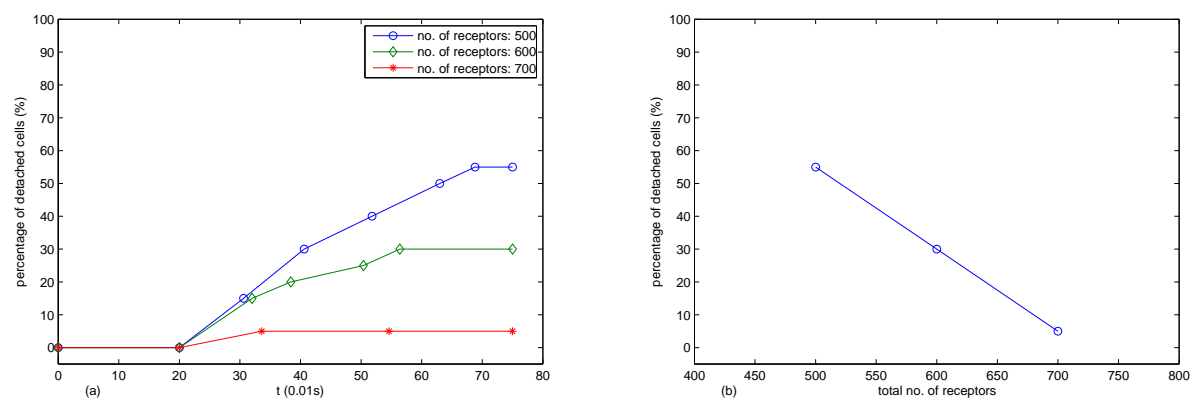

FIG. 5.12. The effect of the total number of receptors per cell on detachment percentage. The figure on the left shows the detachment percentages over time. The figure on the right shows a relationship between detachment percentage and the total no. of receptors.

other things, weakly adhered cells will be swept away in shear flow, as expected.

5.6. The Number of Receptors and Cell Detachment. We studied the influence of the total number of receptors per cell on the cell detachment percentage. We increased the number of receptors per cell from 500, to 600 and 700 . The dynamics of cell detachment over time, from $\mathrm{t}=0$ to $\mathrm{t}=0.75 \mathrm{~s}$, for the shear rate of $80 \mathrm{~s}^{-1}$, is shown in Figure 5.12 left. From these simulations we conclude the following: (1) The larger the number of receptors per cell, the stronger the cell adhesion properties, as expected; (2) The larger the number of receptors per cell, the shorter the time interval until stable adhesion is achieved (cell loss percentage becomes constant). Figure 5.12 right shows the relationship between the percentage of detached cells and the magnitude of the shear rate.

6. Conclusions. We presented a novel multi-scale method and a computational scheme to study cell adhesion and detachment under fluid flow-induced shear stress. The new method couples a kinetics-based cell adhesion algorithm with a fluid-cell interaction algorithm based on the fictitious domain method with distributed Lagrange multipliers. Our method was applied to study the behavior of ear cartilage cells used in lining artificial surfaces of cardiovascular implants. Interesting information was revealed that can be used in modeling and experimental investigation of optimal 
strategies to auricular chondrocytes-coating of cardiovascular implants.

Cell adhesion and interaction with fluid flow embodies a cascade of exceedingly complex phenomena. This work is a gross simplification of reality and should not be considered as a detailed description of the underlying phenomena. However, the multiscale computational algorithm presented here provides an elegant and simple way to capture the main features of cell adhesion under controlled fluid flow conditions, in a way that is within the reach of the today's computational capabilities.

Acknowledgments. We acknowledge the helpful comments and suggestions of G. Guidoboni, R. Bai, E.J. Dean, J. He, H.H. Hu, P.Y. Huang, G.P. Galdi, D.D. Joseph, and Y. Kuznetsov. The authors are particularly grateful to the referees for their careful reading of the manuscript and for their many helpful suggestions which have improved the quality of this work.

\section{REFERENCES}

[1] J. Adams, P. Swarztrauber, and R. Sweet, FISHPAK: A package of Fortran subprograms for the solution of separable elliptic partial differential equations, The National Center for Atmospheric Research, Boulder, CO, 1980.

[2] G.I. Bell, Models for the specific adhesion of cells to cells, Science, 200(1978), pp. 618-627.

[3] M.O. Bristeau, R. Glowinski, and J. Periaux, Numerical methods for the Navier-Stokes equations. Applications to the simulation of compressible and incompressible viscous flow, Computer Physics Reports, 6(1987), pp. 73-187.

[4] S. Canić and D. Rosenstrauch. Use of auricular chondrocytes in lining of artificial surfaces: A mathematical model. IEEE Transactions of Nanobioscience 7(2008), pp. 240-245.

[5] S. Canić, D. Rosenstrauch, Z. Krajcer, K. Ravi-Chandar. Chondrocyte-coated stents for improved biocompatibility: a feasibility study. ABSTR at Symposium on Optimal Stent Design for Cardiovascular Interventions, AAAS Annual Meeting, Seattle, WA, 2004.

[6] K. Chang and D. Hammer, Influence of direction and type of applied force on the detachment of macromolecularly-bound particles from surfaces, Langmuir, 12(1996), pp. 2271-2282.

[7] K. Chang, D.F.J. Tees, and D. Hammer, The state diagram for cell adhesion under flow: leukocyte rolling and firm adhesion , PNAS, 21(2000), pp. 11262-11267.

[8] A.J. Chorin, T.J.R Hughes, J.E. Marsden, M. McCracken, Product Formulas and Numerical Algorithms, Comm. Pure Appl. Math., 31(1978), pp. 205-256.

[9] M. Cohen, E. Klein, B. Geiger, L. Addadi, Organization and adhesive properties of the hyaluronan pericellular coat of chondrocytes and epithelial cells, Biophys J., 85(2003), pp. 1996-2005.

[10] C. Chung, J. Mesa, G. J. Miller, M. A. Randolph, T. J. Gill, J. A. Burdick. Effects of Auricular Chodnrocyte Expansion on Neocartilage Formation in Photocrosslinked Hyaluronic Acid Networks, Tissue Engineering 12(2006), pp. 2665-2673.

[11] E.J. Dean and R. Glowinski, A wave equation approach to the numerical solution of the Navier-Stokes equations for incompressible viscous flow, C.R. Acad. Sci. Paris, Série 1, 325(1997), pp. 783-791.

[12] M. Dembo, D.C. Torney, K. Saxaman, and D. Hammer, The reaction-limited kinetics of membrane to surface adhesion and detachment, Proc. R. Soc. B, 234(1988), pp. 55-83.

[13] C. Dong, J. Cao, E.J. Struble, and H.H. Lipowsky, Mechanics of leukocyte deformation and adhesion to endothelium in shear flow, Ann. Biomed. Eng., 27(1999), pp. 298-312.

[14] C. Dong and X.X. Lei, Biomechanics of cell rolling: shear flow, cell-surface adhesion, and cell deformability, J. Biomech., 33(2000), pp. 35-43.

[15] M. Forero, W. Thomas, C. Bland, L. Nillson, E. Sokurenko, and V. Vogel, Nano Lett. 4(2004), pp. 1593-1596.

[16] R. Glowinski, T.-W. Pan, T. Hesla, and D.D. Joseph, A distributed Lagrange multiplier/fictitious domain method for particulate flows, Int. J. Multiphase Flow, 25(1999), pp. 755-780.

[17] R. Glowinski, T.-W. Pan, T. Hesla, D.D. Joseph, and J. Periaux, A fictitious domain approach to the direct numerical simulation of incompressible viscous flow past moving rigid bodies: Application to particulate flow, J. Comput. Phys., 169(2001), 363-427.

[18] R. Glowinski, T.-W. Pan, and J. Periaux, Distributed Lagrange multiplier methods for 
incompressible flow around moving rigid bodies, Comput. Methods Appl. Mech. Engrg., 151(1998), pp. 181-194.

[19] U.R. Goessler, K.Hörmann, F. Riedel, Tissue engineering with chondrocytes and function of the extracellular matrix (Review), Int. J. Mol. Med., 13(2004), pp. 505-513.

[20] D.A. Hammer and S.M. Apte, Simulation of cell rolling and adhesion on surfaces in shear flow: general results and analysis of selectin-mediated neutrophil adhesion, Biophys J, 63(1992), pp. 35-57.

[21] S. Jadhav, C.D. Eggleton, and K. Konstantopoulos, A 3-D computational model predicts that cell deformation affects selectin-mediated leukocyte rolling, Biophys J, 88(2005), pp. 96-104.

[22] L.H. Juarez, R. Glowinski, T.-W. Pan, Numerical simulation of the sedimentation of rigid bodies in an incompressible viscous fluid by Lagrange multiplier/fictitious domain methods combined with the Taylor-Hood finite element approximation, J. Scientific Computing, 17(2002), pp. 683-694.

[23] M.R. King and D.A. Hammer, Multiparticle adhesive dynamics. Interactions between stably rolling cells, Biophys J, 81(2001), pp. 799-813.

[24] C. Korn, U.S. Schwarz, Efficiency of initiating cell adhesion in hydrodynamic flow, Phys. Rev. Lett., 97(2006), pp. 138103-1 - 138103-4.

[25] S.C. Kuo, D.A. Hammer, D.A. Lauffenburger Simulation of detachment of specifically bound particles from surfaces by shear flow, Biophys J, 73(1997), pp. 517-531.

[26] M.S. Kurtis, T.A. Schmidt, W.D. Bugbee, R.F. Loeser, and R.L. Sah, Integrin-mediated adhesion of human articular chondrocytes to cartilage, Arthritis Rheum., 48(2003), pp. $110-118$.

[27] R.F. Loeser, Integrin-mediated attachment of articular chondrocytes to extracellular matrix proteins, Arthritis Rheum., 36(1993), pp. 1103-1110.

[28] N.A. N'Dri, W. Shyy, and R. Tran-Son-Tay, Computational modeling of cell adhesion and movement using a continuum-kinetics approach, Biophys J, 85(2003), pp. 2273-86.

[29] T.-W. Pan, R. Glowinski, Direct simulation of the motion of neutrally buoyant circular cylinders in plane Poiseuille flow, J. Comput. Phys., 181(2002), pp. 260-279.

[30] T.-W. Pan, R. Glowinski, Direct simulation of the motion of neutrally buoyant balls in a three-dimensional Poiseuille flow, C. R. Mécanique, Acad. Sci. Paris, 333(2005), pp. 884-895.

[31] S. Reboux, G. Richardson and O.E. Jensen Bond tilting and sliding friction in a model of cell adhesion, Proc. R. Soc. A, 464(2008), pp. 447-467.

[32] D. Rosenstrauch, H. Shelat, K. Ravi-Chandar, M. Ng, B. Magesa, A. Tadbiri, D. Paniaqua, D. Fish, S. Canić, OH Frazier. Coating stents with chondrocytes and genetic engineering of auricular chondrocytes. ABSTR Presented at: American Association for the Advancement of Science, 16 Feb 2004.

[33] R.M. Schinagl, M.S. Kurtis, K.D. Ellis, S.Chien, R.L. Sah, Effect of seeding duration on the strength of chondrocyte adhesion to articular cartilage, J. of Orthopaedic Research, 17(1999), pp. 121-129.

[34] T. Scott-Burden, J.P. Bosley, D. Rosenstrauch, K.D. Henderson, F.J. Clubb, H.C. Eichstaedt, K. Eya, I. Gregoric, T.J. Myers, B. Radovancevic, O.H. Frazier, Use of autologous auricular chondrocytes for lining artificial surfaces: a feasibility study, Ann. Thorac. Surg., 73(2002), pp. 1528-1533.

[35] T.A. Springer, Adhesion receptors in the immune system, Nature, 346(1990), pp. 425-434.

[36] M.E. Staben, A.Z. Zinchenko, R.H. Davis Motion of a particle between two parallel plane walls in low-Reynolds-number Poiseuille flow, Phys. Fluid, 15(2003), pp. 1711-1733.

[37] W. Thomas, E. Trintchina, M. Forero, V. Vogel, and E. Sokurenko. Bacterial adhesion to target cells enhanced by shear force. Cell 109(2002), pp. 913-923.

[38] S. Waldman, D. Couto, M. Grynpas, R. Pilliar, R. Kandel. A single application of cyclic loading can accelerate matrix deposition and enhance the properties of tissue-engineered cartilage. Osteoarthritis and Cartilage, 14(2003), pp. 323 - 330.

[39] R. Zaidel-Bar, M. Cohen, L. Addadi, B. Geiger, Hierarchical assembly of cellmatrix adhesion complexes, Biochem. Soc. Trans., 32(2004), pp. 416-420.

[40] E. Zimmerman, B. Geiger, and L. Addadi. Initial Stages of Cell-Matrix Adhesion can be Mediated and Modulated by Cell-Surface Hyaluronan, Biophysical Journal, 82(2002), pp. $1848-1857$. 